\title{
Tissue Engineering-based Therapeutic Strategies for Vocal Fold Repair and Regeneration
}

Linqing Li, ${ }^{1}$ Jeanna M. Stiadle, ${ }^{2,3}$ Hang K. Lau, ${ }^{1}$ Aidan B. Zerdoum, ${ }^{4}$ Xinqiao Jia, ${ }^{1,4,5}$ Susan L.Thibeault ${ }^{2}$, $3^{*}$ and Kristi L. Kiick ${ }^{1,4,5^{*}}$

${ }^{1}$ Department of Materials Science and Engineering, University of Delaware, Newark, DE, 19716, USA;

${ }^{2}$ Division of Otolaryngology-Head and Neck Surgery, Department of Surgery, University of WisconsinMadison, Madison, WI, 53792, USA;

${ }^{3}$ Department of Communication Sciences and Disorders, University of Wisconsin-Madison, Madison, WI, 53792, USA;

${ }^{4}$ Department of Biomedical Engineering, University of Delaware, Newark, DE, 19716, USA;

${ }^{5}$ Delaware Biotechnology Institute, 15 Innovation Way, Newark, DE, 19711, USA

*Corresponding authors: S.L. Thibeault, thibeaul@surgery.wisc.edu and K.L. Kiick, kiick@udel.edu

\begin{abstract}
Vocal folds are soft laryngeal connective tissues with distinct layered structures and complex multicomponent matrix compositions that endow phonatory and respiratory functions. This delicate tissue is easily damaged by various environmental factors and pathological conditions, altering vocal biomechanics and causing debilitating vocal disorders that detrimentally affect the daily lives of suffering individuals. Modern techniques and advanced knowledge of regenerative medicine have led to a deeper understanding of the microstructure, microphysiology, and micropathophysiology of vocal fold tissues. State-of-the-art materials ranging from extracecullar-matrix (ECM)-derived biomaterials to synthetic polymer scaffolds have been proposed for the prevention and treatment of voice disorders including vocal fold scarring and fibrosis. This review intends to provide a thorough overview of current achievements in the field of vocal fold tissue engineering, including the fabrication of injectable biomaterials to mimic in vitro cell microenvironments, novel designs of bioreactors that capture in vivo tissue biomechanics, and establishment of various animal models to characterize the in vivo biocompatibility of these materials. The combination of polymeric scaffolds, cell transplantation, biomechanical stimulation, and delivery of antifibrotic growth factors will lead to successful restoration of functional vocal folds and improved vocal recovery in animal models, facilitating the application of these materials and related methodologies in clinical practice.
\end{abstract}


Key words: vocal folds, lamina propria, tissue engineering, hyaluronic acid, collagen, elastin, resilin, bioreactor, human mesenchymal stem cells, VF fibroblasts, growth factor, regenerative medicine, animal model

\section{Introduction}

The human vocal folds (VF) consist of a pliable vibratory layer of connective tissue called the lamina propria (LP) that controls the production of sound, can sustain up to $30 \%$ strain at frequencies of 100 to $1000 \mathrm{Hertz}(\mathrm{Hz})$, and can reversibly recover after transient stretch to high strain.[1-4] Mechanical stresses, deleterious environmental factors and pathological conditions can disrupt the natural pliability of the vocal folds, resulting in a wide spectrum of voice disorders.[5-7] Voice disorders are the most common communication disorder across the lifespan and dysphonic suffering in individuals causes significant negative influences on their social interactions and daily personal lives.[6] While surgical techniques and behavioral treatments are currently employed to treat voice disorders, surgical interventions can cause scarring and yield inconsistent results. $[5,8]$ Thus, tissue regeneration methods combining the use of bioactive factors, injectable scaffolds, and stem cell therapy have remained of significant research interest in the development of methods to treat vocal fold scarring and vocal fold replacement, $[9,10]$ although challenges in integrated treatment of the mechanical and biological properties of materials has limited the success of these approaches. In this article, we review recent advances and progress in vocal fold repair and regeneration from a tissue engineeringbased therapeutic perspective, focusing on (1) biomaterials intended to mimic the mechanical properties of native VF tissues; (2) bioreactor designs that capture dynamic vocal biomechanical properties, and (3) both in vitro and in vivo applications of these biomaterials, informed by results from bioreactor studies, in vocal fold tissue engineering.

\section{Vocal Fold}

\subsection{Vocal fold scar and societal significance}

Numerous and common stimuli can lead to vocal fold dysfunction and damage, including voice overuse, chemical exposure (e.g., smoke inhalation), gastroesophageal reflux, allergies, intubation, traumas, radiation and inflammation.[7,11,12] Benign vocal fold lesions such as nodules and polyps that can result from these stimuli are usually limited to the SLP, and although temporary relief can be achieved by rest or voice therapy, surgical intervention may be required, 
which can lead to scar formation, reduced flexibility of the LP, and impaired voice production. $[6,12,13]$

Voice disorders are the most common communication disorder across the lifespan and dysphonic suffering in individuals causes significant negative impact on their social interactions and daily lives. There is a paucity of epidemiological data in the literature that is specific to vocal fold scarring and its impact on vocal use. It is, however, well documented that up to $9 \%$ of the general population has some type of voice abnormality and that $29 \%$ of the general population will develop a voice disorder during their life.[8,12,13] Approximately 10\% population of the United States work force involves heavy voice use for their occupation; reaching roughly 28 million people suffering daily voice discomfort causing them to miss work, apply for disability insurance or change occupations. $[8,12-14]$ When costs related to lost work days and treatment expenses, excluding other monetary costs related to pharmaceutical treatment, workman's compensation and change/loss of job are considered, societal expenditures of voice problems in teachers alone has been estimated to be of the order of $\$ 2.5$ billion annually in the United States.[12,15,16] Optimal treatment for voice disorders has not yet been realized, however, and new approaches warrant consideration for regeneration of functional vocal fold tissue.

\subsection{Vocal fold anatomy and composition}

The larynx comprises a number of types of tissue including, but not limited to, cartilage, muscles, epithelium, nerve and stroma and is positioned above the trachea and esophagus. The vocal folds are positioned within the larynx and their primary role is to protect the airway, whereas their secondary role is to produce voice. Arytenoid cartilages adduct and abduct to bring the vocal folds together and apart; the vocal folds are abducted (open) during breathing and adducted (closed) during phonation.[11] The tissues vibrate in a wave-like motion during sound production, $[1,17-20]$ and one of the keys to this mechanical versatility lies in their unique structure and multi-layered composition.

Each vocal fold consists of a stratified squamous epithelium, matrix-rich lamina propria, and thyroarytenoid muscle (Figure 1).[1,10] The epithelium is composed of stratified squamous cells and has a primary role of protecting the deeper layers of tissue, and as a result this layer 
undergoes repeated turnover.[21] The lamina propria (LP) is a collagen and elastin-rich, pliable vibratory layer of connective tissue positioned between epithelium and muscle tissue,[22] subcategorized into three distinct layers: superficial lamina propria (SLP), intermediate lamina propria (ILP), and deep lamina propria (DLP). The outermost layer of the LP, the SLP, sustains the majority of stress during vocal fold vibration.[2] However, these three layers are not distinctly separated to the naked eye in human and animals. The SLP is believed to move freely over the layers beneath it. Deeper layers become increasingly dense with collagen and elastin with the ILP and DLP containing the highest concentration of elastin and collagen.[23,24] The thyroarytenoid muscle is positioned next to the DLP. Vocal fold scar disrupts the mucosal wave, or the movement of the LP, due to increased stiffness and viscosity and can also result in incomplete vocal fold closure.[5] The combined effects of increased stiffness and viscosity cause impaired vibration resulting in voice changes including hoarseness and fatigue.[25,26]

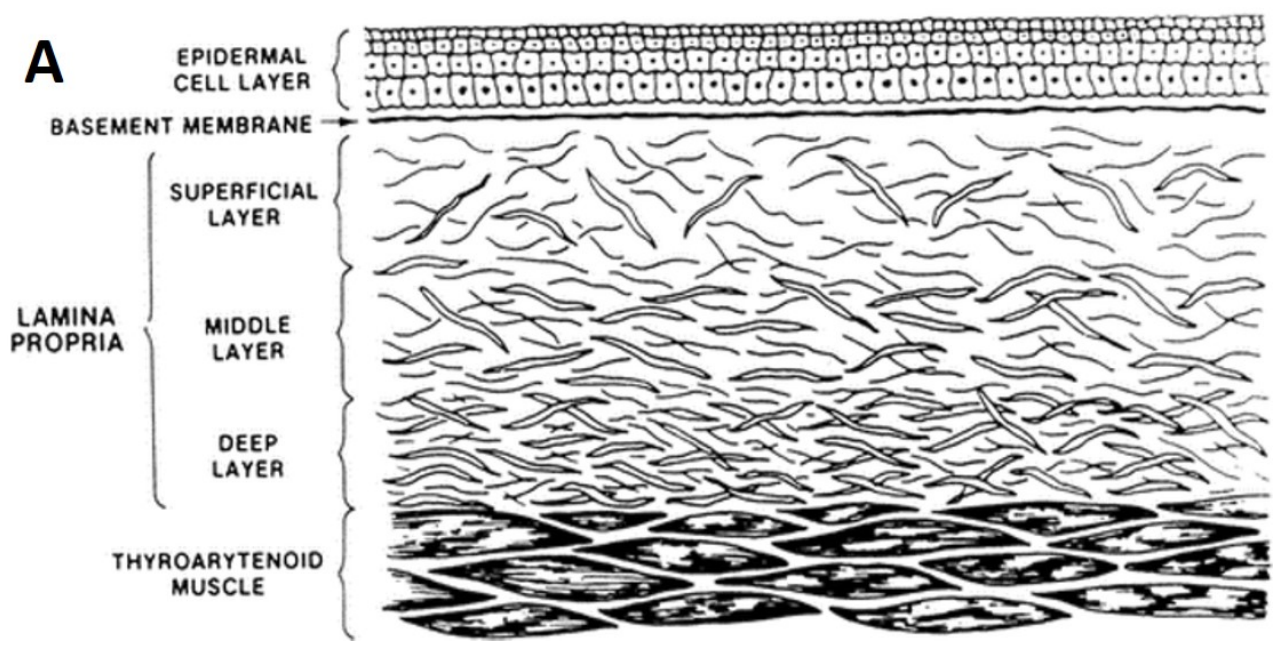



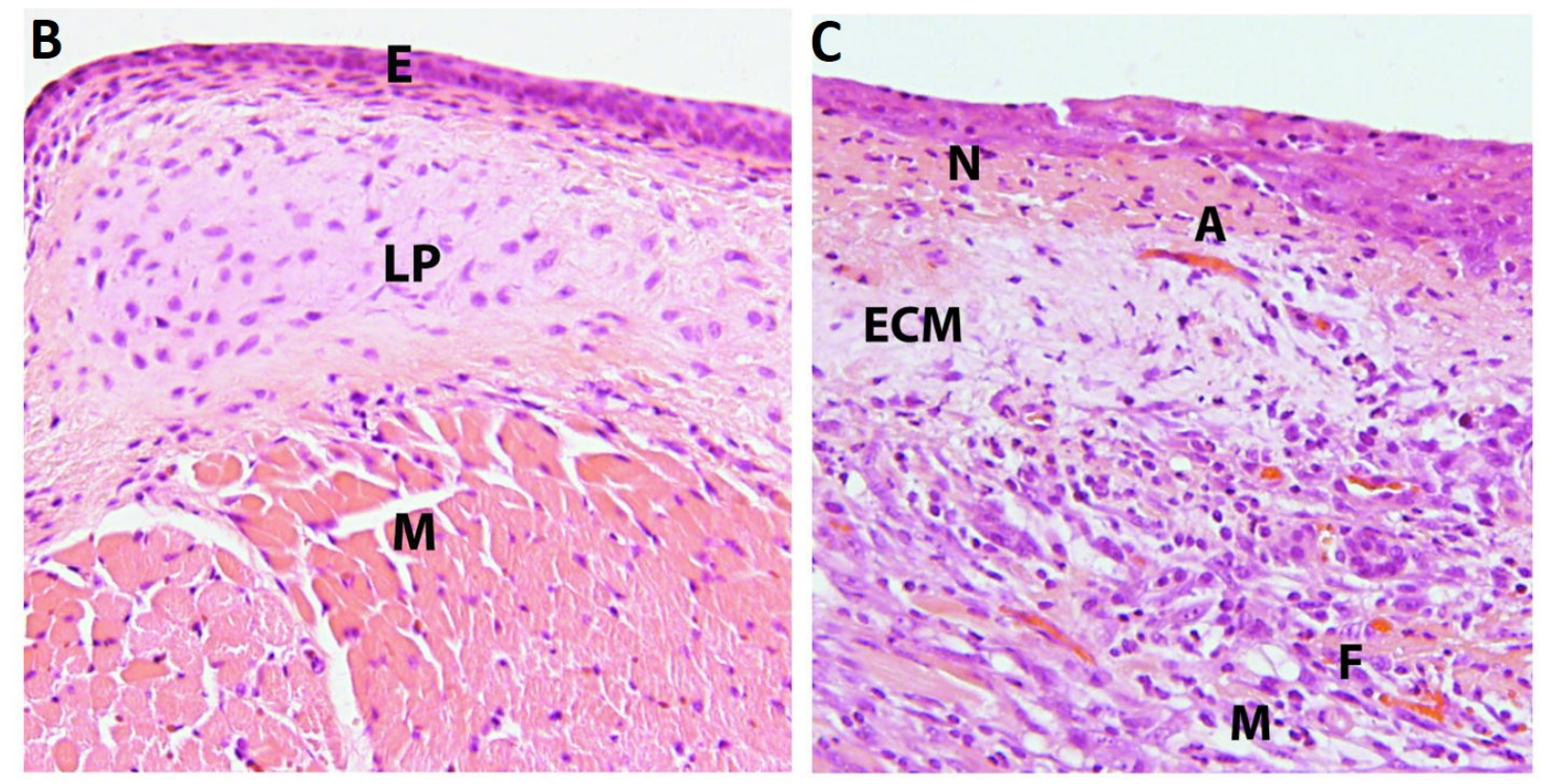

Figure 1. Vocal fold structure. (A) Schematic of the micro-structure of the vocal fold;[27] (B) Coronal histological images of the mid-membranous normal rat vocal fold stained with hematoxylin and eosin (H\&E). Superficial to deep, the layers of the vocal fold include the stratified squamous epithelium (E), the lamina propria (LP) and thyroarytenoid muscle (M); (C) Coronal histological image of the mid-membranous scarred rat vocal fold three days following a scarification injury; the specimen demonstrates the early inflammatory response associated with injury. There is some evidence of granulation, but significant fibrosis has not yet occurred. The epithelium is thick and irregular and the basal cells are dis-organized. The lamina propria is more cellular than found in normal tissue, and contains increased fibroblasts (F) and inflammatory cells including neutrophils $(\mathrm{N})$ and macrophages (M).The extracellular matrix (ECM) also appears to be altered as compared to normal tissues. A few small, thin walled vessels are present which represents angiogenesis (A). [10] Reprinted with permission from Graupp, M.; BachnaRotter, S.; Gerstenberger, C.; et al. Eur. Arch. Otorhinolaryngol. 2015, 1. Copyright (2015) Springer and Bartlett, R. S.; Thibeault, S. L.; Prestwich, G. D., Biomed. Mater. 2012, 7, 24103. Copyright (2012) IOP Publishing.

\subsection{Phonation physiology and mechanical properties of vocal folds}


Vocal folds can be described as two biomechanical layers: the body (thyroarytenoid muscle and DLP) and the cover (epithelium and SLP),[26,28,29] with a main difference between the cover and body layers arising from differences in their oscillation properties. The cover is characterized by its pliability and propagation of oscillatory waves in response to the contraction of the stiff body layer.[30,31] The physiology of phonation that arises from the motion of the vocal folds was first explained by the myoelastic theory of voice production, a theory first developed by Johannes Muller and later modified by Van de Berg.[26] This theory describes the build-up of subglottic air pressure created by the lungs to drive the vocal folds apart. The minimum amount of lung pressure to initiate vocal fold abduction is known as the phonation threshold pressure (PTP).[32,33] In simple terms, the Bernoulli effect causes a drop in pressure in the glottis which, in combination with their elastic properties, serves to drive the vocal folds back together.[26,34] More recently, Titze expanded this theory, demonstrating that the Bernoulli effect is only a minor contributor to the initiation of vocal fold oscillation. Rather, flow induced oscillation allows for sustained oscillation secondary to a continual transfer of energy from the glottal airstream to the tissue, overcoming frictional energy losses in the vocal folds.[33] Mass and stiffness combined with the geometric properties of the vocal folds control self-sustained oscillation. Changes in air pressure across the glottis with convergent and divergent configurations results in asymmetry of air pressures necessary for sustained oscillation. Further comprehensive reviews are recommended for interested readers.[17,33,35]

\section{Current Clinical Approaches and Evaluation of Biomaterials for Vocal Treatment}

Several approaches exist for the treatment of voice disorders including voice therapy, surgery, or some combination of the two. The clinical approach targets for treatment depends on factors such as the pathology's severity and impact as well as the patient's individual needs and motivation for improved voice.

\subsection{Voice therapy and surgical approaches}

Behavioral voice therapy may be offered to balance the respiratory, phonatory, and resonance subsystems, optimize vocal quality, and reduce vocal effort/fatigue. Providing education about vocal hygiene can also assist the patient in management of secondary contributing factors (reflux, hydration, excessive voice use, etc).[6] Currently, there is no research validating a 
specific method of voice therapy for treatment of vocal fold scarring. Behavioral voice therapy may be implemented as a preventative measure to limit further damage to the scarred tissue and voice quality. The patient may develop compensatory hyperfunctional behaviors (e.g. ventricular/anterior posterior compression) due to the stiffened tissue from vocal fold scar and resultant changes to voice quality. Benninger and colleagues suggest that engaging in voice therapy may significantly improve a patient's voice quality even after scar maturation.[5] Different therapy approaches may be recommended depending on the nature of the patient's pathology as well as the patient's concerns and motivation[26,36]. For example, a resonant voice approach (such as the Lessac-Madsen approach) targeting a specific laryngeal configuration may be useful to reduce both respiratory effort and impact of vocal fold vibration while achieving clearer voice quality.[11] Titze et al. suggested semi-occluded voice exercises (e.g., lip trills, humming, straw phonation) as a way to create sound more efficiently with less impact/tissue collision.[37] Confidential voice may serve to reduce hyper-functional behaviors by emphasizing quiet, breathy productions.

Surgical approaches have focused on either altering the lamina propria or correcting glottal insufficiency. Injections of biomaterials or steroids have been used as a treatment for mildmoderate vocal fold scar, by targeting the SLP to restore some vibratory characteristics of the tissue.[38] However, many of these materials are absorbed into the tissue over time, whereby repeated injections are required.[39] Medialization thyroplasty may be used to repair glottal insuffiency without directly contacting the lamina propria.[40] In addition, Hoy et al. described the use of an ultra-fast laser for ablation vocal fold scar tissue.[41] Welham et al. investigated the use of surgical treatments for vocal fold scar in a prospective clinical study, where they found that the thyroplasty and graft implantation methods resulted in reduced vocal handicap scores of patients with vocal fold scar, but no improvement in other assessment measures.[42] However, injection laryngoplasty resulted in no improvement in any of the outcome measures.

\subsection{Choice of animal models and clinical materials}

Many types of animal models have been proposed to study vocal fold biology, treatment of vocal fold injury and scarring. The type of animal model used depends on the nature of the experiment and outcomes of interest. One advantage to using animal models to study wound-healing of the 
vocal folds is that animals may be sacrificed and larynges excised to perform histological and other assays examining the ECM. Commonly used species include mouse, rat, rabbit, pig, and canine. Bless and Welham reported the characteristics of the vocal ligament, ECM, biomechanical environment, and animal's vocalizations as important considerations for animal models of vocal fold scars.[43] For example, a mouse model may be advantageous for manipulating gene expression, but the small size of their larynges can be considered a limitation. Tateya et al. describe the advantages of a rat model for vocal wound healing including its short life span, small cost, quick wound-healing process, and similarities of its lamina propria to that of humans.[44] Thibeault and colleagues investigated the histological and rheological characterizations in a rabbit vocal fold model. They discovered the structure of the lamina propria in the rabbit is similar to that of a human vocal fold in the arrangement of multiple lamina propria layers containing collagen, elastin, and HA and thyroarytenoid muscle.[25] A canine model has also been suggested by Rousseau and colleagues as a relevant model for vocal fold wound-healing studies due to the size of the lamina propria, ability to phonate, and the amount of tissue it provides.[45] Woodson investigated the use of a porcine model to study vocal fold scar. Advantages include the similar size and structure of porcine vocal folds compared to human vocal folds.[46]

To date, different biomaterials have been used in vocal fold injection and implantation to restore vibratory characteristics and/or medialize the vocal fold edge damaged by scar formation. Types of materials used have evolved to include a wide range of options for surgeons to use. Teflon was historically used as a more permanent material for vocal fold augmentation, but has been associated with undesirable foreign-body reactions.[40] More commonly used materials for injection for vocal fold medialization include Cymetra (collagen-based), Radiesse (calcium hydroxyapatite), and autologous fat.[40] Sataloff described autologous fat as an effective implantation for improving mucosal wave in patients with severe scar formation.[36,37] Pitman et al. investigated the use of temporalis fascia transplantation for treatment of vocal fold scar and sulcus in a group of 10 patients.[45] They found that one year after surgery there was improvement for the majority in patient-reported measures ( 9 out of 10 patients), perceptual measures ( 7 out of 10 patients), and laryngeal videostroboscopy ( 9 out of 10 patients). However, there was no statistically significant improvement in acoustic measures. 
Lott and Janus reviewed the various surgical approaches of tissue engineering for vocal fold scar including development of synthetic and biologic scaffolds, cell therapies, and bioactive factors (e.g., growth factors).[46] Promising cell therapies include the use of fibroblasts, embryonic stem cells and mesenchymal stem cells for treatment of vocal fold scar. Fishman and colleagues reviewed the results of a variety of stem cell therapy strategies in animal models. They described the use of both endogenous cell sources including side population cells, stellate cells, and human vocal fold fibroblasts as well as exogenous cell sources such as MSCs, ASCs, and myoblasts. The main conclusion from this review was that although many promising approaches exist, most of these have only been tested as a means of treating acute injuries rather than chronic vocal fold scar. They emphasized the need for clinical trials investigating their use in human models.[47]

The foremost reason for the inability to effectively address vocal fold scarring is that current surgical options disrupt the biomechanical properties of native tissues, often causing secondary scar formation, and injectable hydrogels do not capture the complex tissue composition of the vocal fold ECM. To date, only specific growth factors are administered in clinical settings for treatment of scarred or atrophied vocal folds, mostly antifibrotic growth factors that are known to prevent scar formation. In 2009, Hirano and colleagues conducted the very first human trial on a 63-year-old male, who was treated with basic fibroblast growth factor (bFGF) for vocal fold scar and showed improvements in clinical features only after 1 week of injection.[47-49] Another strong antifibrotic potency hepatocyte growth factor (HGF) has also demonstrated significant improvements in mucosal wave amplitude, glottal incompetence and phase asymmetry via injecting HGF-containing hydrogels to scarred vocal fold tissues in in vivo canine animal models.[50-52] Although these grow factor treatments showed superior improvements compared to saline controls, complete restoration of vibratory properties and anatomical microstructures has not yet been achieved, in addition to the issue regarding safety concern of potential tumorigenicity.[9,53] Therefore, integration of such strategy with tissue engineered scaffolds for controlled delivery of growth factors in vivo and implementation of simultaneous multipotent cell delivery can achieve an optimal clinical outcome; materials developed to address these challenges would offer unique opportunities. 


\subsection{Promise of materials approaches}

Materials-based approaches that would either ameliorate effects of scarring and/or yield tissueengineered substitutes offer compelling alternatives in the treatment of vocal fold scarring.[9,54] Key concerns in such approaches include: (1) control of the mechanical properties of the biomaterial, (2) inclusion of appropriate biological cues, (3) application of appropriate mechanical stimuli to guide appropriate ECM development, and (4) control of the degradation of the biomaterial on length- and timescales commensurate with new tissue growth.[54-60] Recent generations of polymer/polypeptide-based biomaterials have been designed to control cell binding, growth factor release, and/or cell-mediated degradation in efforts to address the concerns above.[54,55,57,61-64] However, there are very few synthetic materials that contain the necessary combination of mechanical properties, growth factor delivery profiles, and degradation profiles for optimal generation of vocal fold tissue; this arises in large part from synthetic difficulties in the production of such complicated materials. In addition, approaches to characterize materials and culture cells under mechanically relevant conditions have lagged far behind their demonstrated need. Specific advances in each of these areas are delineated below.

\section{Biomaterials in Development/Research}

Based upon the fundamental tissue engineering principles, injectable, bioactive and biodegradable polymers and hydrogels with appropriate chemical composition, structure characteristics, and tailored mechanical properties have been extensively employed for addressing voice disorders.[10] This approach, from a clinical perspective, offers attractive advantages over traditional materials implantation, as it has the potential to solidify injecting materials into any desired shape at the site of injury with enhanced interfacial interlocking with surrounding normal tissues. This strategy also minimizes the invasiveness and potential trauma to patients, limits the risk of infection and secondary scar formation, and reduces treatment costs with easy practice and increased patient accessibility.[10,65] To date, injectable hydrogels investigated for vocal fold tissue regeneration have largely focused on hyaluraonic acid (HA) and its derivatives due to the significant relevance of HA's biological and biomechanical functionalities in vivo. [65,66]

\subsection{Hyaluronic-acid-based materials}


Hyaluronic acid (HA), a linear non-sulfated and negatively charged glycosaminoglycan, is a major ECM component of SLP with roughly $6.4 \mu \mathrm{g}$ mass for each mg of total protein in the vocal fold LP.[67] This high concentration of HA combined with its unique structural properties contributes significantly to its physiological, biological and mechanical properties in vivo. Specifically, the largely extended, loosely coiled molecular structure of HA allows extensive hydrogen bonding with water to yield highly viscous solutions, and thus it functions as a shock absorber to resist tissue compression where it acts as a tissue damper to protect the edges of vocal folds from oscillatory trauma encountered during sound production.[65,68] Furthermore, the osmotic, viscoelastic and space-filling features of HA are critical in phonation as these properties influence the thickness, shape, and viscosity of the vocal fold.[68,69] HA also interacts with cells through multiple cell surface receptors (e.g. CD44, present on the surface of VFFs and MSCs) to control signal pathways and regulate the HA degradation and mediate the inflammatory response (e.g., inhibit macrophage migration and aggregation).[70] Unfortunately, native HA is often quickly degraded (within 3-5 days) after injecting into the VF through local hyaluronidase-mediated enzymatic degradation.[71,72] This rapid degradation profile is undesirable from a tissue engineering perspective. Thus chemical modifications of HA are required to generate HA-derivatives with various reactive groups through carboxylate or hydroxyl residue conjugations (Figure 2) that rapidly crosslink to form hydrogels with improved mechanical stability, controlled scaffold degradation, and elongated material retention in vivo.

Previous attempts to treat vocal fold scarring either by directly treating the SLP or with injection laryngoplasty have utilized augmentative substances such as polytetrafluoroethylene (Teflon), silicon, bovine collagen, and fat. However, observation of various drawbacks including inflammation, migration, immunogenic reaction, and resorption required search for other native substances such as HA-based hydrogels. A divinyl sulfone crosslinked HA hydrogel Hylan-B (also known as Hylaform and clinically available for plastic surgery for intradermal implantations), shown to be non-immunogenic, non-toxic and non-inflammatory in animal models, was proposed to treat vocal fold glottal insufficiency.[73] In 2002, Hylan-B gel was employed in a prospective and randomized clinical trial in Sweden, where Hylan-B and bovine collagen were injected to 83 patients with glottal insufficiency, and outcomes were examined at 1, 6 and 12 months post-injection. Evaluations were judged by patients' subjective ratings (e.g., 
visual analogue scales) coupled with digitized videostroboscopic measurements, phonetograms, and maximum phonation time.[74] Twelve months after injections, patients' self-ratings suggested significant improvements in both groups supported by the improved glottal closure characterized by videostroboscopy. Interestingly, the Hylan-B gel group exhibited preservation of the vibration amplitude and glottal area variations, and exhibited less resorption and increased maximum phonation time compared to collagen-injected VFs.[74] To further elucidate the differences observed in vivo, various augmentation substances were injected into non-injured rabbit vocal folds, and the mechanical properties (e.g., viscoelasticity) were compared to the native rabbit VF tissues. All injected materials exhibited decreasing dynamic viscoelastic properties with increasing oscillatory frequency. However, Hylan-B gel was the only injection group that reached similar viscosity to non-injected control samples and yielded the lowest dynamic viscosity over vocal folds injected with Teflon or crosslinked collagen (Zyplast) in both short-term and long-term conditions.[75,76] Further expansion to repair damaged vocal folds involved the modification of Hylan-B gel and the introduction of photo-polymerizable groups in order to adjust the swelling ratio and fine-tune the degradation kinetics of methacrylated HA hydrogels.[77] To date, Hylan-B gel and its derivatives were only used as space filling materials instead of as cell-gel constructs for comprehensive therapeutic treatment, and unfortunately Hylan-B is currently no longer on the market for clinical use in the United States.

Alternatively, a disulfide cross-linking strategy was developed to form soft hydrogels with thiolmodified HA in order to achieve improved material flexibilities and tunable hydrogel properties.[78] Dithiobis(propanoic dihydrazide) (DTP) was coupled to HA via carbodiimide chemistry to yield HA-DTPH macromolecules (Figure 2) that can be oxidized to form disulfide linkage-based HA hydrogel. The formation of disulfide bond was reversible and hydrogels could be degraded upon the addition of dithiothreitol (DTT) or in the presence of hyaluronidase (HAse). The mild gelation reaction of the HA-DTPH solution under physiological conditions enabled the encapsulation of L-929 murine fibroblasts and supported the cell viability and proliferation in vitro.[78] Thiolated-HA films were implanted into subcutaneous flank and peritoneal cavity in a Wistar rat model and subsequent histological and cytochemical analysis revealed only a mild inflammatory response at both sites of implantation after 42 days in 
vivo.[79] Unfortunately, the clinical application of this material is limited due to relatively long gelation time required to form stable hydrogels.

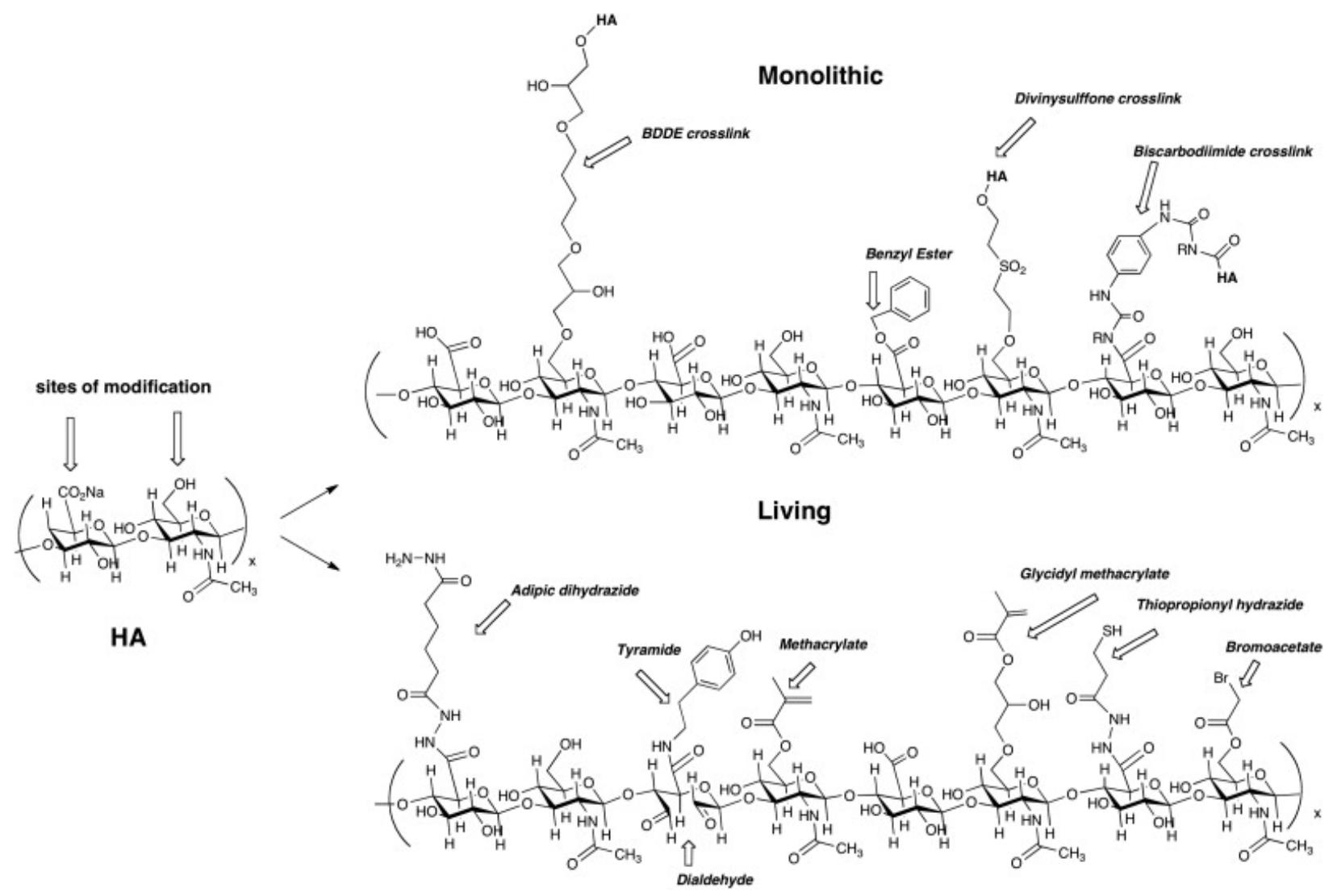

Figure 2. Sample chemical structures of monolithic (top) and living (bottom) chemical modifications of hyaluronic acid. A hypothetical composite structure illustrates selected primary modifications: adipic dihydrazide for use in further crosslinking via acrylamide or hydrazone linkages; butane-1,4-diol diglycidyl ether, a prototypical monolithic crosslinker for HA; tyramide for peroxidase crosslinking; dialdehyde obtained by periodate oxidation; methacrylate on primary 6-hydroxyl group; benzyl ester; glycidyl methacrylate; thiopropionyl hydrazide from DTPH modification; bromoacetate; an unmodified disaccharide unit for comparison.[66] Reprinted with permission from Burdick, J. A. and Prestwich, G. D. Adv. Mater. (2011) 23, H41.Copyright (2011) Wiley.

With improvements in fitting material parameters and crosslinking reactions to a narrow range of physiologically tolerant temperatures and aqueous environment, in situ crosslinkable and injectable hydrogels expanded their use in surgical implantation and clinical application. 
However, challenges remain in addressing the slow hydrogel gelation kinetics that potentially compromise the encapsulation and delivery of stem cells for in vivo vocal fold injection. In order to promote fast gelation, polyethylene glycol diacrylate (PEGDA, Mw=3400) was incorporated into HA-DTPH hydrogel system, with fast gelation ( $t<9$ mins) achieved by tuning the molar ratio of PEGDA and HA-DTPH.[80] Materials properties such as porosity, crosslinking density, gelation kinetics, swelling ratio and degradation can be easily modulated via changing the material compositions (e.g., molar ratio between PEGDA and HA-DTPH). In vitro cytocompatibility was confirmed via encapsulating T31 human tracheal scar fibroblasts in these hydrogels with results indicating 10-fold increase in cell density after 4-week culture. In vivo biocompatibility was evaluated via subcutaneously implanting cell-loaded hydrogels in the flanks of nude mice. Immunohistochemical staining results indicated that encapsulated cells maintained the fibroblast phenotype and secreted extracellular cytokines without inducing necrosis or damage to surrounding tissues.[80]

Further modification of the carboxylic acids on the HA backbone via carbodiimide-mediated hydrazine chemistry to generate reactive thiol groups was achieved in order to alter the viscosity and reduce the degradation rate of a new class of HA derivative, Carbylan-S, also known as CMHA-S (Figure 3). To determine whether the application of injectable HA-based hydrogels at the time of intentional VF resection could facilitate wound repair and preserve the unique viscoelastic properties of the vocal fold ECM, prophylactic injection of Carbylan-SX hydrogel (Carbylan-S crosslinked with PEGDA) was performed and compared to HA-DTPH+PEGDA hydrogel and saline injection in a rabbit model. Three weeks after injection and biopsy, the Carbylan-SX-treated vocal folds exhibited significantly less fibrosis formation compared to those of saline-treated scarred tissues and showed improved biomechanical properties of viscosity and shear elastic moduli values compared to HA-DTPH-PEGDA-injected vocal fold tissue (Figure 4).[81] These findings demonstrated that prophylactic in vivo manipulation of the extracellular matrix with an injectable Carbylan-SX hydrogel is capable of inducing vocal fold tissue regeneration to yield optimal tissue composition and biomechanical properties favorable for phonation, as well as minimize vocal fold scar formation at the time of surgery. 


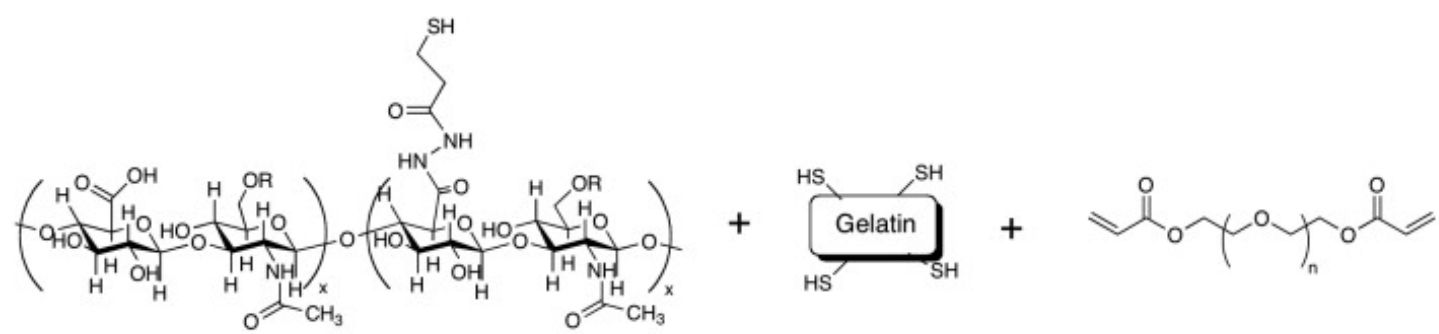

Thiol-modified HA (Glycosil)

Gelatin-DTPH (Gelin-S)

PEGDA (Extralink)

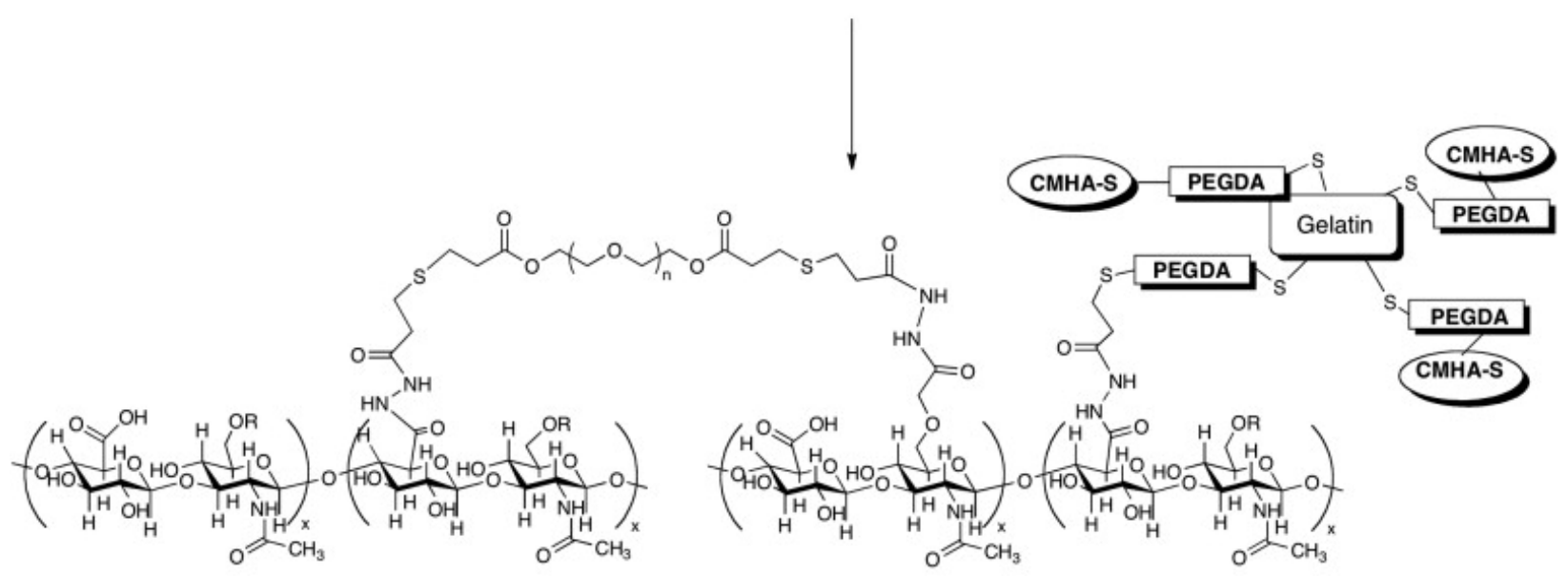

Extracel sECM

Figure 3. A thioether crosslinked semi-synthetic ECM formed by crosslinking thiol-modified carboxymethyl HA (CMHA-S) with thiol-modified gelatin using the bifunctional crosslinker, PEGDA.[66] Reprinted with permission from Burdick, J. A. and Prestwich, G. D. Adv. Mater. (2011) 23, H41. Copyright (2011) Wiley.
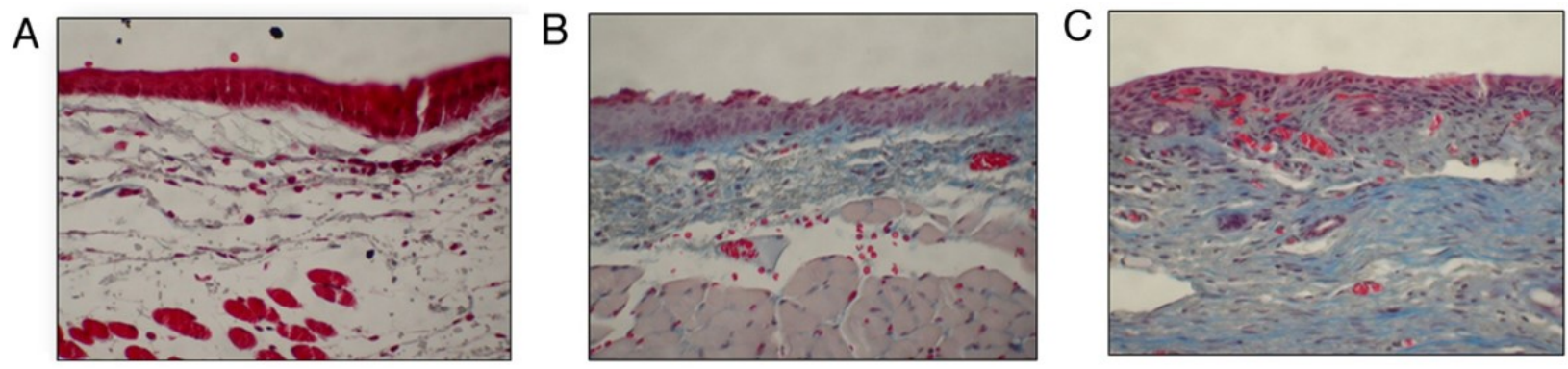

Figure 4. Representative coronal sections 40x of the vocal fold treated with a trichrome stain. Statistical significance established by a blinded pathologist qualitatively categorizing the fibrosis level for each section. (A) Carbylan-SX treated vocal folds showing mild fibrosis. Visual inspection indicates a significant decrease in fibrosis between the Carbylan-SX treatment group and saline-treated controls $(\mathrm{p}=0.0158)$; (B) HA-DTPH-PEGDA treated vocal folds showing 
moderate fibrosis. No statistical difference between fibrosis levels observed in saline-treated controls $(\mathrm{p}=0.1645)$; (C) Saline treated controls showing moderate fibrosis.[65] Reprinted with permission from Gaston, J. and Thibeault, S. L. Biomatter (2013) 3, e23799. Copyright (2013) Taylor \& Francis Group.

In order to improve cell attachment in HA-based injectable hydrogels towards a vocal fold tissue engineering application, a cell-adhesive, thiolated derivative of gelatin (Gtn-DTPH, 3,3'dithiolbis(propanoic hydrazide)-modified gelatin) was introduced and co-crosslinked with Carbylan-S and PEGDA via a Michael-type addition reaction to form stable viscoelastic gels designated as Carbylan-GSX or Extracel ${ }^{\circledR}$ (Figure 3). Mongeau and coworkers measured the viscoelastic properties of Carbylan-GSX hydrogels over a wide range of high frequencies (40$4000 \mathrm{~Hz}$ ) using a Rayleigh wave propagation method, and the storage and loss moduli were easily modulated by tuning gelatin concentrations to be within the range of mechanics of human VF tissues obtained from in vivo and ex vivo measurements.[82-84] The Thibeault group evaluated in vitro cytocompatibility of this matrix by culturing an immortalized human vocal fold fibroblast (hVFF) cell line in two-dimensional and three-dimensional conditions and cell viability, morphology, proliferation, apoptosis, transcript gene expression, and inflammatory markers were measured. In 2D culturing system, immortalized hVFFs developed typically stretched, spindle-like fibroblast morphology similar to those seeded on the surface of polystyrene. In contrast, 3D encapsulated hVFFs in Carbylan-GSX hydrogels exhibited rounded, isolated morphology similar to those observed in Matrigel (Figure 5).[85] No significant differences were observed in cell viability, morphology, proliferation, apoptosis, and cell marker protein expression between Carbylan-GSX and Matrigel after 7 days of cell culture. Transcript gene analysis suggested similar expression of fibromodulin, transforming growth factor $\beta$-1 (TGF $\beta-1)$, and tumor necrosis factor-alpha (TNF- $\alpha$ ) between Garbylan-GSX and Matrigel. However, up-regulation of fibronectin, hyaluronidase 1 and cyclooxygenase II and downregulation of interleukins 6 and 8, hyaluronic acid synthase 3 and Col I of hVFFs in CarbylanGSX hydrogels were confirmed.[85] Later, the role of TNF- $\alpha$ in wound repair was systematically studied on a 3D, hVFF-encapsulated Carbylan-GSX hydrogel containing a range of dosages of TNF- $\alpha$ with results indicating that injectable hydrogels can enhance the role of TNF- $\alpha$ in promoting VF ECM degradation, remodeling the lamina propria, and potentially accelerating the 
wound healing process.[86] Cumulatively, these investigations proved that Carbylan-GSX did not induce significant toxicity or inflammation with additional secretion of mRNA relevant to the ECM constituents existing in lamina propria tissue.

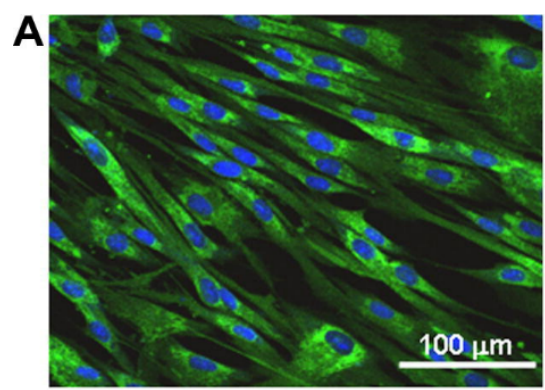

polystyrene

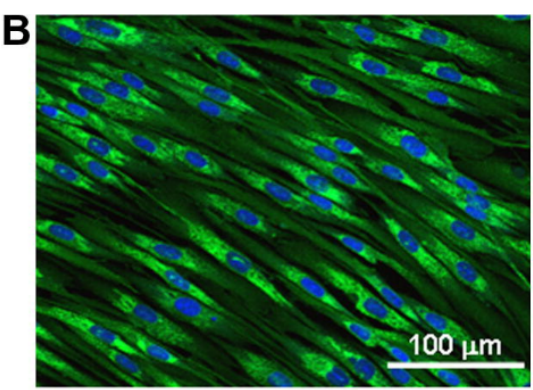

2D Carbylan GSX

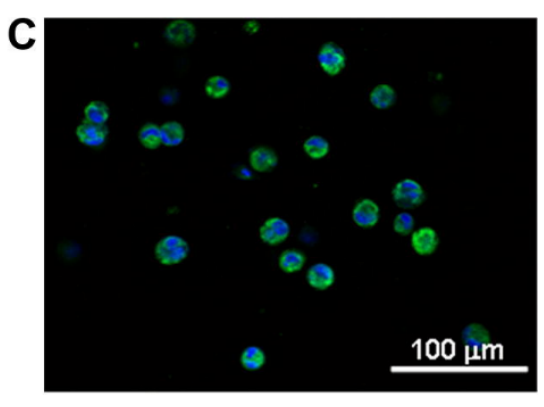

3D Carbylan GSX

Figure 5. Morphologic appearance and fibroblast marker (hPH) distribution of immortalized hVFFs in various culture environments. After $72 \mathrm{~h}$ of in vitro culture, fluorescent images of hVFFs on polystyrene (A), on a thin layer of Carbylan-GSX (2-D condition) or in Carbylan-GSX (3-D condition) were captured by an inverted confocal microscope equipped with dual excitation lasers for green and blue fluorescence.[85] Reprint with permission from Chen, X. and Thibeault, S. L. Acta Biomater. 2010, 6, 2940. Copyright (2010) Elsevier.

In initial in vivo applications, Carbylan-GSX with various gelatin concentrations was unilaterally injected in a rabbit vocal fold wound healing model to determine the optimal material composition that would promote wound repair and induce VF tissue regeneration.[87] The biomechanical properties and tissue repair outcome were also compared to those injected with Carbylan-SX hydrogels and saline controls. Gene expression analysis 3 weeks post operation revealed a higher level of HAse 2 production for Carbylan-SX and Carbylan-GSX 20\% (20\% gelatin) compared to saline control. Saline control samples demonstrated elevated viscous and elastic moduli compared to both Carbylan-SX and Carbylan-GSX gels due to higher level of fibrosis and unorganized collagen deposition. Carbylan-GSX containing 5\% gelatin exhibited analogous biomechanical properties to human vocal fold mucosa and improved biomechanical properties to the greatest extent among all gelatin concentrations. $[68,83,88]$ 
The following investigations used this optimized material composition, Carbylan-GSX 5\%, to characterize the molecular response during early stage wound repair in the vocal fold tissue for functional tissue improvements and implementation of future regenerative medicine strategies.[89] Twenty rabbits underwent bilateral vocal fold biopsy followed by immediate injection of Carbylan-GSX 5\% into the left vocal fold with saline injection to the right; larynges were harvested at days 1, 3, 5 and 10 post-surgery and real-time reverse transcriptase polymerase chain reaction was employed to measure transcript expression of selected markers. At day 5, significant expression differences were found in procollagen, fibronectin and transforming growth factor $\beta 1$ (TGF- $\beta 1$ ) between Carbylan-GSX $5 \%$-treated and saline-treated groups, indicating the importance in establishing the initial wound structure to provide a basic lattice for subsequent healing events and to improve wound healing. Results indicated that sECMs in the wound bed during early stage tissue repair can amplify the normal rabbit vocal fold woundhealing process over a short period of time which may lead to the recovery of functional biomechanical properties of VF tissues required for voice production.[89] Long term effects of prophylactic hydrogel injections on tissue repair and biomechanics of lamina propria in a rabbit model was also conducted 6 months after treatment. Carbylan-GSX 5\%-treated rabbit vocal folds exhibited viscous properties and elastic shear moduli values significantly lower than salineinjected scar tissue.[72] The improved viscoelastic properties of rabbit VFs measured at 6 months post-treatment is likely due to improved healing processes with minimal fibrosis formation. These results indicated that the early benefits of prophylactic injection of HA-derived hydrogels at the time of surgery (measured at 21 days post-injury) could be maintained through to the chronic stage of repair after 6 months, suggesting the utility of injectable hydrogels in facilitating vocal wound repair and engineering scarred vocal fold tissue.

In parallel, various biomimetic approaches have also been investigated towards tissue regeneration of the scarred vocal fold lamina propria using HA-based hydrogels.[90,91] The therapeutic effectiveness and outcome have been evaluated in injured rat vocal fold model among following groups: (1) transplantation of isolated bone marrow mesenchymal stem cells (BM-MSCs) alone, (2) injection of Carbylan-GSX 5\% hydrogel alone and (3) delivery of Carbylan-GSX 5\% hydrogel with encapsulated BM-MSCs.[91] The results indicated that treatment of vocal scarring with BM-MSC injected in a Carbylan-GSX hydrogel exhibited the 
most favorable outcomes in promoting ECM deposition, hyaluronan metabolism, and production of TGF- $\beta 1$ without increasing myofibroblast differentiation. This combined delivery approach displayed no detectable cytotoxicity and preserved local cell proliferation, thus enhancing the qualities and biological competence of the replace tissues. Therefore, embedding BM-MSCs or any other relevant cell types in a vehicle of degradable HA-based hydrogels for injection in injured vocal folds represents an attractive therapeutic strategy for future clinical trials.

\subsection{Native materials and biocomposites}

ECM-derived native materials, such as collagen and fibrin, can be reconstituted in vitro to form mechanically stable bioactive matrices for 3D cell culture scaffolds and fundamental biology investigations. Decellularized tissue scaffolds These native ECM-based tissue engineering scaffolds have thus been extensively studied and applied to various biomedical applications due to their complex structural integrity and function, biological recognitions, and inherent biocompatibility.[54,56,92] Kanemaru et al. was the first to report the therapeutic benefit of injecting autologous MSCs encapsulated in 1\% hydrochloric acid atelocollagen to facilitate the regeneration of injured canine vocal folds.[93] The observed in vivo cell proliferation within the atelocollagen scaffold indicated that atelocollagen is suitable for vocal fold tissue engineering. Kishimoto et al. continued to investigate the restorative effects of a scaffold on vocal fold scarring and sulcus vocalis by implanting atelocollagen sheet into patients with post-cordectomy scar or sulcus vocalis. This improved atelocollagen sheet is biocompatible and biodegradable with sufficient porosity for cell infiltration and prolonged residence time in vivo for cell-guided ECM remodeling within injured lamina propria tissue.[94,95]

Long et al proposed that replacing the entire vocal fold cover instead of addressing only the lamina propria might be a more effective strategy to treat severe VF scars. They initially encapsulated adipose-derived stem cells in fibrin hydrogels and applied epidermal growth factor (EGF) which led to the differentiation of ASCs into epithelial cells near the air surface interface with the majority of mensenchymal cells remaining undifferentiated within the bulk matrix. The resulting stratified tissue-engineered bilayered constructs resemble the vocal fold epithelium and lamina propria.[96] Further mechanical characterization revealed that the epithelialized construct shared similar elastic indentation moduli and collagen microstructure with excised human VF 
covers (Figure 6). The construct was also able to oscillate and withstand physiological shear airflow when attached to an excised larynx model of phonation, suggesting that replacement of the entire vocal fold cover might provide another alternative to effectively address severe VF scars.[97]

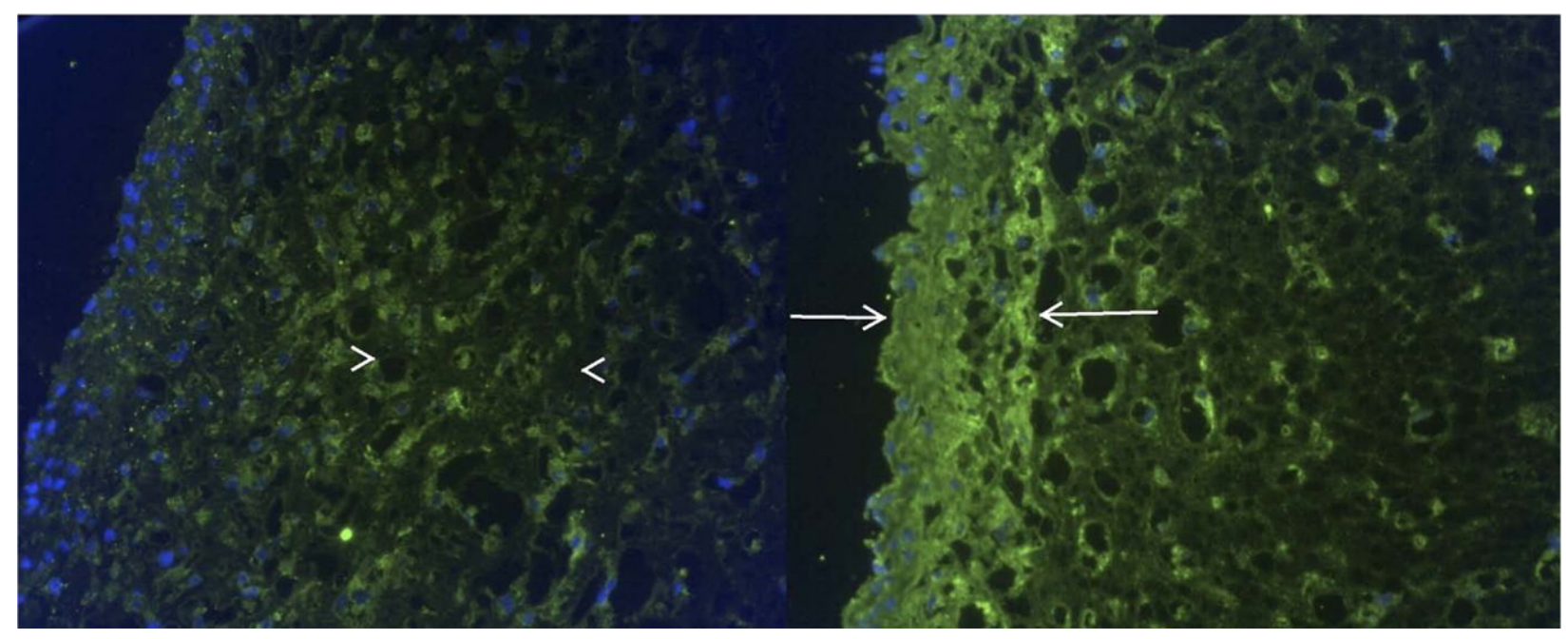

Figure 6. Immunohistochemical labeling for type I collagen in fibrin-ASC tissue constructs. Bilayered construct on the left shows green collagen labeling in the middle segment (arrowheads). Homogeneous construct on the right shows intense labeling near the surface (arrows). In both, nuclei are labeled blue. (Original magnification: 20x)[97] Reprint with permission from Long, J. L.; Neubauer, J.; Zhang, Z.; et al. Otolaryngol.- Head Neck Surg. 2010, 142, 438. Copyright (2010) SAGE Publishing.

Although collagen, fibrin and other naturally-derived materials described above have previously resulted in some level of improvement in VF function post-implantation, gel resorption and/or compaction has generally limited the long term in vivo outcome of these materials. For example, previous collagen injections intended to remediate VF scarring have been reported to yield diminutive outcomes due to unmatched biomechanical properties of collagen (a dynamic viscosity is an order of magnitude higher than normal VFs)[98] as well as foreign body reaction and resorption response in vivo.[5,99] Most recently, ground-breaking studies from the Welham group describe fabrication of a type-I collagen-based organotypic model where patient-donor isolated primary vocal fold fibroblasts (VFF) were encapsulated with epithelial cells and subsequently seeded at the air-liquid interface of the matrix to create a transplantable, 
bioengineered VF mucosa.[100] These approaches offer potential for treatment of patients with severe VF fibrosis and large tissue loss. The resulting bioengineered-mucosae exhibited encouraging resemblance in morphology to native human VF mucosa at 2 weeks with a distinguished $\sim 50 \mu \mathrm{m}$-think stratified squamous epithelium layer on top of sparsely VFFpopulated lamina propria. Furthermore, this VFF-VFE co-cultured platform also demonstrated proteome-level evidence of organogenesis-specific enrichment coupled with emerging ECM protein complexity, anatomic substructural architecture and initiation of immature physiological epithelial barrier function. The VFF-driven contraction of this bioengineered mucosa was able to sustain physiological driving pressures, restore high-frequency vibratory function with physiological mucosal wave travel and aerodynamic-to-acoustic output in an ex vivo excised canine larynx model. The translational therapeutic efficacy of the bioengineered VF mucosa was confirmed by auto- and allo-engraftments into humanized mice in vivo, exhibiting low immunogenicity and high tolerance in the human adaptive immune system.[100] This elegant bioengineering tissue regeneration framework offers significant potential for producing biochemically capable, physiologically relevant and clinically valuable bioengineered VF mucosae. It lays the groundwork for future investigation in mechanical force/signal input and the design of structured scaffolds, such as introducing fibrous proteins to capture the layered architecture of native VF mucosa. Towards this end, in order to determine the optimal composition of synthetic ECMs that would promote the wound healing and tissue regeneration for vocal fold tissue engineering without scar formation, natural biomaterials are often combined with other naturally abundant biomacromolecules to achieve desired material properties and enhanced biocompatibility.

The combination of natural macromolecules has been actively employed as a novel strategy to enhance matrix complexity and to generate composite materials that can encompass a wide range of physical and chemical properties to accommodate a broader spectrum of functional requirements.[101,102] By optimizing material composition between two or more components in a given system, desired composite materials can be fabricated as particles, films, fibers and hydrogels.[103-108] These platforms offer material analogues comparable to synthetic polymers, yet with expanded utility from the tailorabilities of material composition, mechanical properties, degradation kinetics, chemical functionalization, and intrinsic biocompatibility. 
Applications include vocal fold tissue engineering, but also drug delivery, biosensors, and electrical and optical devices.

Zeitels and coworkers investigated in vitro effects of 5 hydrogels (HA, collagen, fibrin, fibrincollagen, and fibrin-HA) on the differentiation of adipose-derived stem cells (ASCs) to establish the long-term conditions necessary for controlling the differentiation of ASCs into equivalent counterparts of functional SLP fibroblasts.[109] Proliferation data suggested cells encapsulated in fibrin-based hydrogels (fibrin, fibrin-collagen and fibrin-HA) exhibited higher growth rates and elastin expression compared with cells grown in collagen or HA gels, possibly due to the stabilization of scaffolds by increasing compaction and cell-cell contact within the fibrin gel consistent with previous reports.[110] ASCs encapsulated in the composite hydrogels displayed elongated morphology, secreted the decorin marker, and demonstrated GAG synthesis, indicating ASC differentiation. The data presented suggested possibilities of controlling stem cell differentiation via choosing appropriate scaffolds for VF tissue engineering applications. Hahn et al combined collagen with either HA or a GAG-analog, alginate to investigate their ability to support the ECM synthesis and to inhibit VFF-mediated hydrogel compaction. The results revealed that injectable collagen-alginate hydrogels were able to support the viability of 3D encapsulated VFFs and stimulate the development of spindle-shaped cell morphology and vocal fold relevant ECM synthesis with limited observation of matrix compaction over 42 days of culture compared to collagen-HA hydrogels, suggesting the potential as VF SLP regeneration scaffolds.[104]

Other HA biocomposite hydrogels involved mixing human adipose-derived MSCs (hAdMSCs), HA with mildly cross-linked alginate hydrogel as an injectable cell carrier to promote VF repair. The existence of mildly-crosslinked alginate improved the residence of HA in vivo without leading to any abnormal tissue response in a wound healing model. The incorporation of hAdMSCs in HA-alginate hydrogel elongated the retention and maintained the viability of hAdMSCs in vivo up to 1 month in a VF-injured rabbit model.[111] Histological evaluation suggested that the administration of hAdMSCs in HA-alginate hydrogels ameliorated excessive deposition of collagen I, up-regulated the HGF activity in regenerating VFs, and exhibited functional improvements in restoration of VF mechanics compared to only hAdMSCs treated 
injured VFs, likely due to the stimulation of HGF activity of native fibroblasts. The data suggested the great potential of hAdMSC-containing HA-alginate hydrogel towards promoting the residence of stem cells in VFs and facilitating VF wound healing without fibrosis.[112]

Composite hydrogels based on hydrazide-modified HA and aldehyde-modified dextran with various crosslinking densities were also investigated for augmentation/regeneration of the lamina propria tissues in ferret VF.[113] The results demonstrated that the in vitro degradation kinetics and mechanical properties can be modulated via adjusting the proportions of these two polysaccharides. With the increased dextran content, the crosslinking density increases, which directly increases the mechanical properties and decreases the degradation rate. Mild inflammatory response was observed around material-tissue interfaces with less crosslinked HAdextran composite hydrogel formulation in vocal folds using a ferret animal model, suggesting the promise of in vivo application of HA-dextran-based composites.[113] Although these ECMderived natural biocomposite materials exhibited promising results toward regenerating injured or atrophied VF tissues, they inevitably suffer from batch to batch variation, heterogeneous chemical composition, poor mechanical properties, and lack of flexibility in independent tuning of scaffold properties, which limits the application of these materials for engineering the complex, layered structure of human VFs. Descriptions of decellularized tissue scaffolds are not included in this review, due to the fact that these types of materials do not offer the chemical versatility of synthetic materials for engineering structural, mechanical and biological properties, which are the focus of this review. For details on the use of decellularized materials, interested readers are referred to other publications.[114-116] 

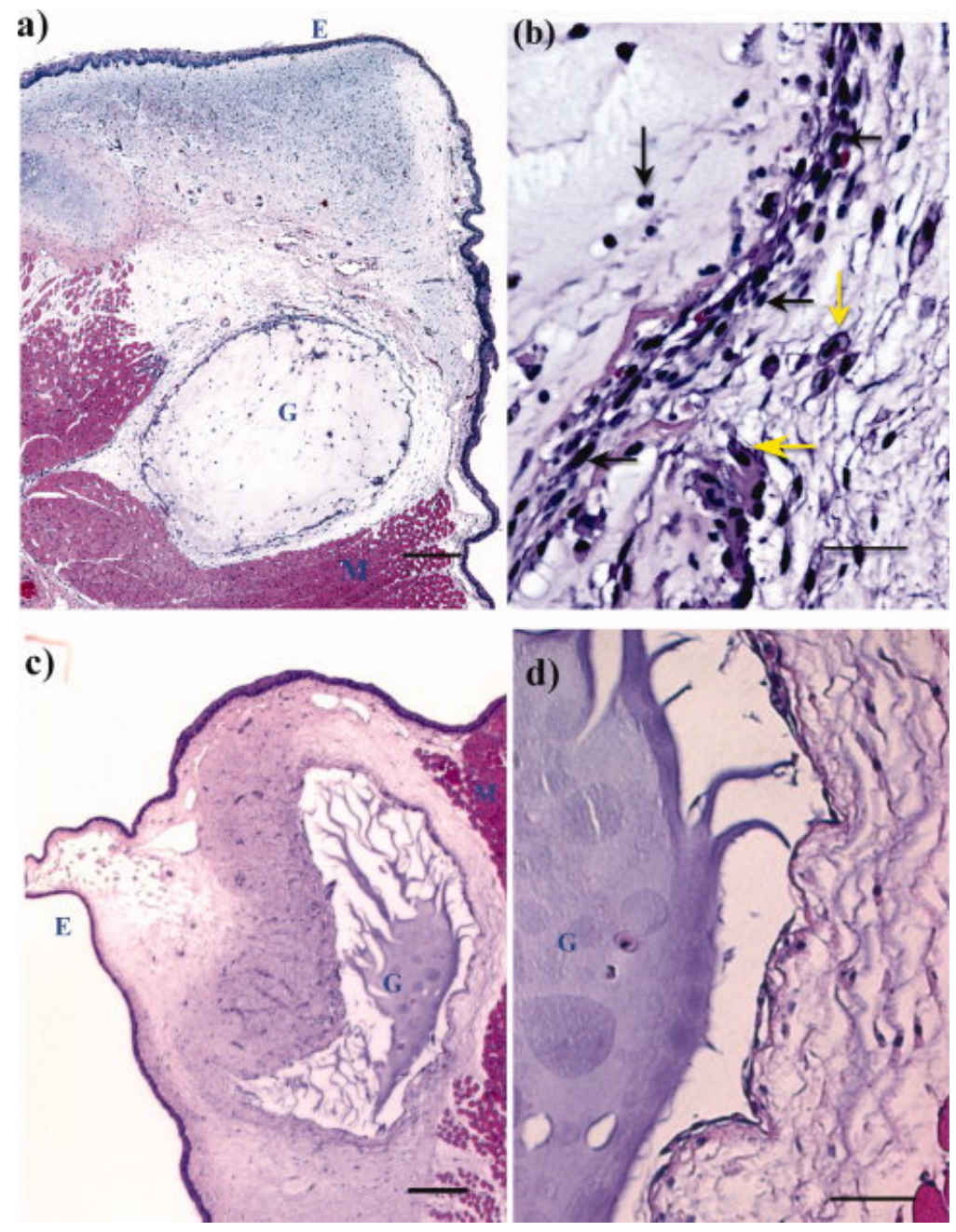

Figure 7. H\&E stained tissue slides of HA-Dextran crosslinked hydrogel implanted in the ferret vocal fold at week 3. Regions of epithelium, injected gel and muscle tissue are indicated by letters E, G, and M, respectively. (a) HA-dex0.75 in the host tissue (scale bar: $200 \mu \mathrm{m}$ ); (b), the interface of the host tissue and HA-dex0.75 gel (scale bar: 50 $\mathrm{m}$ ); (c), HA-dex1 in the host tissue (scale bar: $200 \mu \mathrm{m}$ ); (d) the interface of the host tissue and HA-dex1 gel (scale bar: $50 \mu \mathrm{m}$ ). Cell types were visually identified in $\mathrm{b}$ and pointed by arrows (yellow horizontal arrow: macrophage; yellow vertical arrow: foam cell; black vertical arrow: neutrophil; black horizontal arrows: fibroblasts).[113] Reprint with permission from Luo, Y.; Kobler, J. B.; Heaton, J. T.; et al. J. Biomed. Mater. Res. B. Appl. Biomater. 2010, 93, 386. Copyright (2010) Wiley.

\subsection{Microgels}

The aforementioned simple mixture of various natural biopolymers generally yields scaffolds with compromised structural integrity and poor mechanical stability. Although chemical 
crosslinking reactions have been introduced in those systems to improve mechanical properties and degradation time, the limited surface contact area between natural biopolymers inevitably hampers the desired microstructure and mechanics of a biomaterial. In attempts to overcome these limitations, biocompatible microgels with large surface-to-volume ratio and wide range of particle size distributions have been investigated for numerous biomedical applications including vocal fold tissue engineering.[117-119] Jia et al. developed HA-based microgels and crosslinked microgel networks with tunable degradation and mechanical properties suitable for vocal fold regeneration. Hydrazide-modified HA (HAADH) and aldehyde-functionalized HA (HAALD) were crosslinked to form microgels through the inverse emulsion droplets yielding microspheres with an average diameter size of $10 \mu \mathrm{m}$. The presence of residual functional groups allows subsequent cross-linking of the microgels with other polymers to generate doubly crosslinked networks $(\mathrm{DXN}) \mathrm{s}$. DXNs had tunable viscoelasticity similar to the range of canine vocal fold tissue when assessed via torsional wave experiments measured at human phonation frequencies.[120] The group modified their strategy and adopted a more versatile technique for preparing crosslinkable HA hydrogel particles (HGP) via chemical crosslinking with divinyl sulfone using a sodium bis(2-ethylhexyl) sulfosuccinate (AOT)/isooctane/water reverse micelle system to fabricate particles with reduced size $(\mathrm{d} \sim 900 \mathrm{~nm})$. Sodium periodate-oxidized HA HGP can be further crosslinked with HAADH to form macroscopic hydrogels with two distinct hierarchical micro-networks; one within individual particle gels and another among different particles. The microstructural properties of HA-DXNs were characterized by microscopic and neutron scattering techniques with images indicating two distinct phases. (Figure 8) These HADXNs microgels exhibited highly pliable extensibility (strain-to-break approx. 200-300\%) and similar elastic shear moduli to those of the vocal fold lamina propria. In vitro cell proliferation assays revealed that these microgel-reinforced DXNs did not elicit adverse effects on the viability and proliferation of fibroblasts, indicating the potential application of HA-DXN hydrogels for regeneration of the vocal fold layered mucosal microstructures.[121,122] 

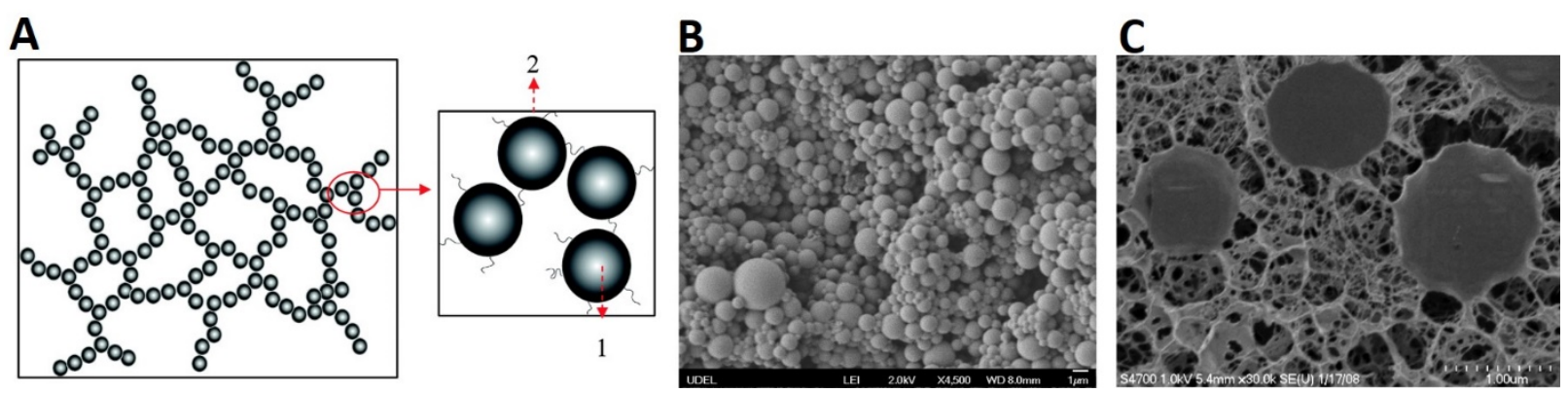

Figure 8. Schematic representation of doubly cross-linked networks (DXNs). (A) HA-based doubly crosslinked networks with (1) intra-particle cross-linking between DVS and HA and (2) inter-particle cross-linking between oxHGPs and HAADH; (B) Scanning electron micrograph of HA HGPs; (C) CryoSEM images of doubly cross-linked networks.[122] Reprint with permission from Jha, A. K.; Hule, R. A.; Jiao, T.; et al. Macromolecules 2009, 42, 537. Copyright (2009) American Chemical Society.

The Mongeau group fabricated a HA-Gtn-based, hierarchical network structure with microparticles embedded and crosslinked in a secondary HA-based network to reinforce mechanical properties, with an application aimed towards vocal fold injection. The composite microgels were successfully fabricated through copolymerization of HA, Gtn and PEGDA using a standard "water in oil" emulsion technique yielding uniformly distributed particles ( $\sim 800 \mathrm{~nm}$ diameter) in HA-networks. The particles' Young's modulus were $\sim 22 \mathrm{kPa}$ and bulk gel shear moduli were around $75 \mathrm{~Pa}$ at $1 \mathrm{~Hz}$, as characterized via AFM and oscillatory rheology measurements respectively.[123] These microparticle-reinforced hydrogels promoted hVFF adhesion, spreading, proliferation and exerted enhanced cell migration (average motility speed: $0.24 \mu \mathrm{m} / \mathrm{min}$ ) in the external HA network as a result of controlling cellular adhesion at the interface of densely-crosslinked microgels, a phenomenon not observed in HA-Gtn composites lacking microgels.[124]

In vivo vocal fold injection of HA-Gtn-based microgels for the treatment of acute vocal fold injury was investigated in Sprague-Dawley rats following unilateral vocal fold stripping. Saline, HA-bulk gel and HA-Gtn microgel were injected into the LP 5 days after surgery and H\&E staining as well as other immunohistochemistry staining for macrophages, myofibroblats, elastin, collagen type I and III were analyzed. The results indicated that the macrophage count was significantly lower in the HA-Gtn microgel group than that in the saline-injected VFs at day 28, 
suggesting that HA-Gtn microgels did not induce a significant inflammatory response.[125] These composite hydrogels with enhanced surface areas that allow improved cell adhesion and migration offer an effective cell substrate platform for potential therapeutic avenue for VF regeneration.

\subsection{Synthetic polymer materials}

Current surgical techniques and standard augmentation substances have proven rather difficult for the complete treatment of $\mathrm{VF}$ scars or paralysis, with clinical management procedures producing inconsistent and often suboptimal results.[5,126,127] The encouraging outcomes discussed in the aforementioned in vivo animal experiments and initial human trials of injectable biomaterials proposed to treat VF scars may not be uniformly useful, due to the heterogeneity of voice disorders and the limited tunability of the properties of the most commonly employed materials (e.g., viscoelasticity, procedural simplicity). Polymer-based materials have been widely employed for various biomedical applications including vocal fold tissue engineering due to their flexibility in tuning materials properties such as porosity, crosslinking chemistry and density, microstructures and mechanical strength, as well as coupling multiple biological moieties to the scaffolds.[56,63,128-130]

Hahn and coworkers employed a US Food and Drug Administration (FDA) approved polymer, polyethylene glycol (PEG)-based hydrogel (PEG30) to investigate the effects of functional material parameters (e.g., mesh size, mechanics, degradation rate and bioactivity) on cell behavior and pliability of the mucosa. Porcine VFFs were encapsulated in four PEGDA hydrogels with degradation half-lives of approximately 25 days with results indicating initial elastic compressive moduli and mesh sizes ranging from $30-100 \mathrm{kPa}$ and $9-27 \mathrm{~nm}$, respectively.[131] After 30 days of in vitro culture, VFF ECM deposition and phenotype demonstrated a strong correlation with initial hydrogel mesh size and elastic moduli. Specifically, sulfated glycosaminoglycan (sGAG) synthesis increased with the increased initial modulus and the decreased mesh size, while elastin production decreased with these parameters. The collagen deposition strongly depended on initial hydrogel mesh size, which is consistent with the trend of myofibroblastic phenotype induction of encapsulated VFFs.[131] Zeitels and coworkers further investigated in vivo biocompatibility by injecting PEG30 hydrogel unilaterally 
into 16 normal canine VFs and subsequently evaluated the vocal fold function by recording vibration and phonation threshold pressures using high-speed video and analyzing 3-dimensional reconstruction using magnetic resonance images (MRI) and histological staining.[132] To ensure maximal impact on VF vibratory function, 11.7-T MRI imaging serial-section histological analysis of excised confirmed that PEG30 hydrogels were injected into the superficial layer of the VFs (Figure 9). All PEG30-injected VFs displayed mucosal wave activity with low average phonation threshold pressure, indicating that the presence of PEG30 did not have a detrimental impact on normal phonatory vibration, vibratory amplitude and phonation threshold pressure compared to the non-injected, contralateral vocal folds.[132] Only a minimal biological response was observed in the SLP with no evidence of an acute inflammatory response either in terms of the vocal fold surface or histological findings. Time-dependent resorption of PEG30 hydrogel by phagocytosis yielded minimal foreign body reaction without inducing fibrosis or causing mechanical stiffness. Overall, the data presented a promising biocompatible candidate to restore functions to deficient phonatory mucosa with additional modulation of material properties toward VF clinical application.[132]
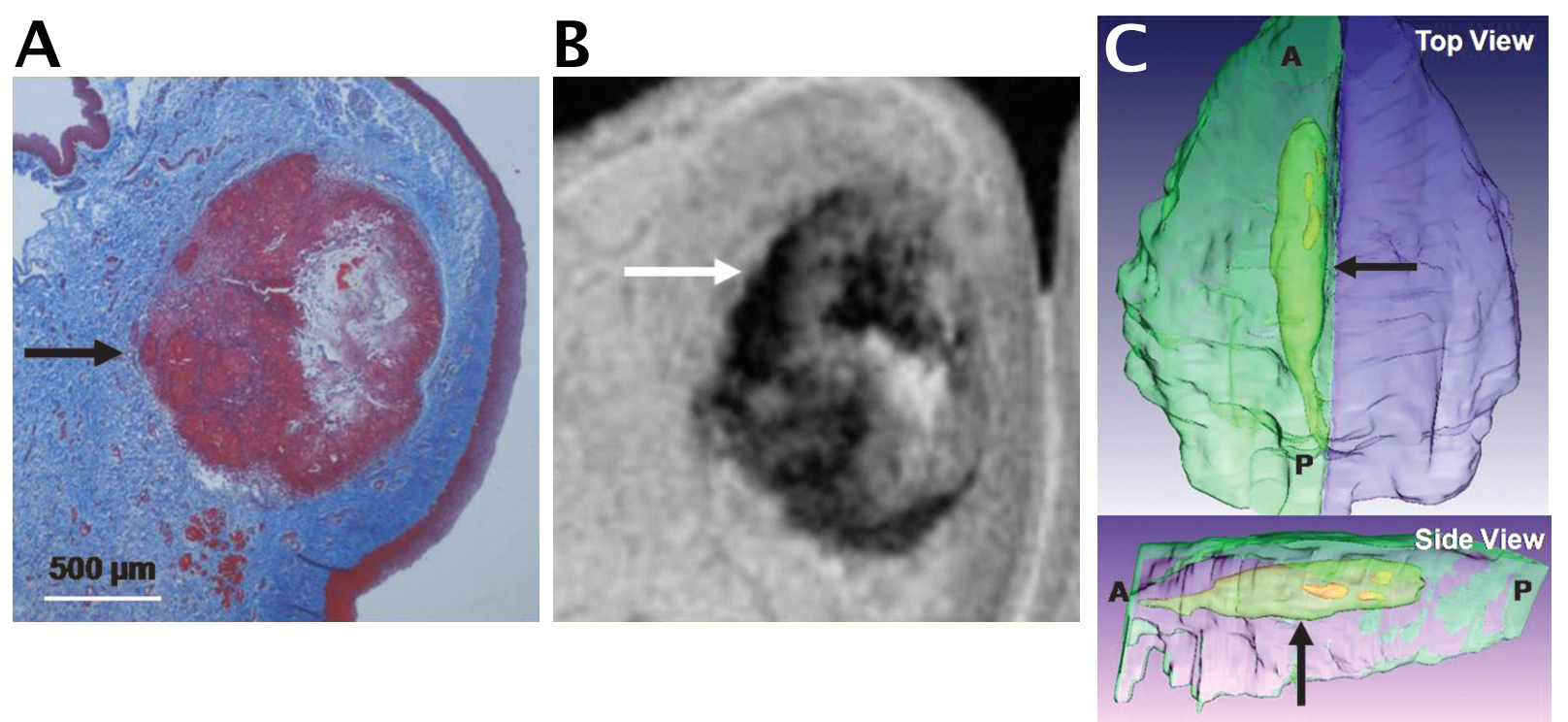

Figure 9. Representative example of histology-magnetic resonance imaging matching (A: histology and B: MRI) that was used for 3-dimensional reconstruction of vocal fold with Amira software (C). Arrows show location of residual PEG30 and cellular infiltrate. A: anterior; P: posterior.[132] Reprint with permission from Karajanagi, S. S.; Lopez-Guerra, G.; Park, H.; et al. Ann. Otol. Rhinol. Laryngol. 2011, 120, 175. Copyright (2011) SAGE Publications. 
Kwon and coworkers selected other FDA approved polymers (polycaprolactone (PCL) and Pluronic F127) and developed injectable hydrogels with PCL spheres embedded within thermoresponsive Pluronic F127 matrix for injection laryngoplasty in a rabbit model to evaluate its potential to treat unilateral vocal fold palsy. Endoscopic and histological analysis showed that the PCL spheres/Pluronic F127 gel mixture exhibited excellent integration with the surrounding tissues, maintained the injected volume without migration or induction of inflammatory response, decreased the vocal gap, and improved asymmetric VF movement, suggesting its potential as a new therapeutic biomaterial to treat VF paralysis.[133] In parallel, the authors investigated the effects of another synthetic and biodegradable polymer poly(lactic-co-glycolic acid) (PLGA) with Pluronic F127 mixture for the augmentation of atrophied VFs. Laryngoscopic analysis showed that both $5 \mathrm{wt} \%$ and $10 \mathrm{wt} \%$ PLGA/Pluronic F127 gels maintained their integrity 8 weeks after injection without inducing significant inflammatory response. High-speed camera examination revealed regular and symmetric collision of VF mucosa without distorted movement, confirming its suitability for injection and offering potential additional advantages of incorporating growth factors or delivering drugs in the vocal folds.[134]

Other polymer-based matrices have also recently studied and reported for vocal fold tissue engineering application. Polyether polyurethanes (PEUs), a commercially available medicalgrade polymer, were proposed as a 3-dimensional environment where candidate cell types and therapeutics would be seeded and vibrated in a bioreactor to evaluate the effects of stress, strain and frequency on cellular behavior.[135] The morphological and mechanical properties of various concentrations of PEU foams with different porosities were characterized and compared to native VF tissues. Oscillatory rheology, dynamic mechanical analysis and scanning electron microscopy data revealed that the tunable elastomeric mechanical properties of PEUs (shear storage moduli in the range of $2-6 \mathrm{kPa}$; shear loss moduli from $0.2-0.62 \mathrm{kPa}$ ) with $20-\mu \mathrm{m}$ diameter pore size are suitable for cell seeding. The authors proposed that the range of PEU mechanical properties can serve as a platform for modeling various VF diseases, such as vocal scarring or different phases of mucosal hydration.[135] Together, these examples suggest that the integration of synthetic versatility, tunability of desirable bulk mechanical properties, and 
controlled matrix degradation of polymeric biomaterials could offer great potential in optimizing material properties for vocal fold tissue engineering.

\subsection{Polypeptide-based materials}

\subsubsection{Elastin-like polypeptides}

Elastin is the second most abundant protein in vocal folds. Thin, mature elastin fibers comprise approximately $9 \%$ of total protein by weight, and play a pivotal role in vocal fold vibration.[67] Previous quantitative analysis of elastin distribution in the LP revealed that mature elastin fibers allows LP tissues to extend to large deformation and recoil to its original configuration repeatedly under high frequency. The superficial layer of the lamina propria contains a considerable amount of immature elastin fibers, while high concentrations of mature elastin fibers exist in the intermediate and deep layers of LP.[67,136] Changes in the formation, structure and organization during elastin fiber self-assembly can alter vocal fold vibration and mucosal wave propagation, compromising the critical mechanical properties of VF (e.g., viscosity, elasticity) and potentially interfering with the production of sound.[2,136-138]

Although the vocal fold contains a larger amount of elastin than that found in skin, native elastin is rarely used in tissue engineering scaffolds due to its substantial insolubility, extraction difficulty, the need for high temperature and multi-step purification, and the potential of disease transmission.[139-141] Given native elastin's remarkable properties including significant elasticity, self-assembly features, mechanical stability, and biological activity, the generation of various types of synthetic elastin-based biomaterials is attractive.[140,142] Accordingly, over the past two decades, tremendous research efforts have been devoted to the synthesis of "biorubbers" that mimic the inherent elasticity of natural elastin. Recombinant DNA technologies and solid-phase peptide synthetic methodologies have been applied for the synthesis of artificial tropoelastin, elastin-based peptides and elastin-like polypeptides and fabrication of elastin-based hydrogels, micelles, and electrospun fibrous scaffolds, with essentially most studies employing amino acid sequences based on the consensus sequence of elastin, VPGVG.[107,143-149]

Motivated by the mechanical properties and biological features of natural elastin in native vocal fold tissues, Grieshaber et al developed elastin-based hybrid elastomeric hydrogels that mimic the multiblock molecular architecture of tropoelastin and the excellent mechanical properties of 
native elastin.[150] The multiblock elastin-mimetic hybrid polymers (EMHPs) are composed of alternating poly (ethylene glycol) block and alanine-rich, lysine-containing elastin-peptide block synthesized via azido-alkyne mediated orthogonal click reaction.[151,152] EMHPs can be chemically crosslinked with hexamethylene diisocyanate (HMDI) to form elastomeric hydrogels with compressive modulus close to $0.12 \mathrm{MPa}$ when hydrated and without exhibiting any deleterious effects to the growth of primary porcine vocal fold fibroblasts (PVFFs).[150] Following the initial success of the synthesis of EMHPs, a cell-binding domain (CBD) was chemically conjugated to the backbone of this polymer-peptide multiblock and the crosslinked EMHP-CBDs supported the attachment, spreading and proliferation of neonatal human dermal fibroblasts (NHDFs).[153] Inspired by the highly aligned and organized ECM network (which becomes disorganized upon injury) in normal vocal folds,[2,22] the Woodhouse and Thibeault groups investigated the impact of aligned and unaligned fibers on cellular morphology, gene expression, and matrix organization of vocal fold fibroblasts on electrospun, ELP4-coated TecoflexTM scaffolds for the development of potential VFLP construct candidates.[154] This ELP4 contains the VGCAPG-based 20-24-24-24-24 gene sequence with exon 21 and 23 as crosslinking domains between the hydrophobic domains.[155,156] The ELP4 coating was introduced to the electrospun scaffolds in order to enhance the biological function and promote ECM deposition during wound healing in scarred vocal fold tissues. Both unaligned and aligned scaffolds with or without coated ELP4 promoted viability and proliferation of HVFFs with aligned scaffolds demonstrated a significant effect on the angle of orientation of seeded HVFFs. However, with the ELP4 coating, aligned fibrous scaffolds up-regulated elastin synthesis after 7 days of culturing without a concomitant up-regulation of collagen III synthesis.[154] Collectively, these results indicate that the elastin-coated, aligned scaffolds are promising candidates and could provide significant insights to guide vocal fold scar treatment.

Overall, these elastin-inspired biomaterials demonstrate encouraging in vitro findings with VFLP-related cell types, provide interesting clinical implications and offer possible insights to the generation of novel implants and strategies for treating vocal fold scars and disorders. However, the multiple steps in the chemical synthesis, the limited tunability of material parameters, and the mismatch of the mechanical properties of the resulting materials with those of native VFs have largely minimized the applications of elastin-based biomaterials towards 
vocal fold tissue engineering, with most materials showing significant hysteresis upon application and release of strain and limited independent tuning of biological properties. Other types of "bio-rubbers" that are totally or partially synthetic do not offer the complexity of their natural counterparts. Thus, the development of new elastomeric biomaterials would offer new opportunities in VF treatments.

\subsubsection{Resilin-like polypeptides}

Another novel bio-rubber, the insect structural protein resilin, which is normally found in the specialized compartments of the cuticles of insects, offers unique and unprecedented advantages in engineering new elastomeric and highly mechanically active biomaterials.[157,158] Resilin exhibits rubber-like elasticity characterized by low stiffness, high extensibility, and efficient energy storage, which support its critical roles in insect flight and jumping.[159-164] Like elastin, resilin contains distinct, repetitive domains of elastic sequences, and once crosslinked, resilin exhibits excellent elastic properties, with extremely high rubber efficiency (resilience).[165,166] Resilin also exhibits a remarkable fatigue lifetime, surviving repeated contraction/extension cycles, in some cases greater than 400 million cycles, of 2004000Hz.[142,167-169] Resilin isolated from insect sources is a functional elastomer at frequencies up to at least $25 \mathrm{~Hz}$, where its resilience is still $70 \%$, and it is a major component of the wing systems of insects with wing-beat frequencies in this range.[142,170-172] Although this protein was discovered over 50 years ago,[159,173-175] its application had been severely hampered by the lack of reliable routes for its large-scale production. The excellent reversible elasticity, high-frequency responsiveness, and hydrophilicity of natural resilin motivated our interests to expand the use of RLPs as a new class of bioelastomers in the engineering of mechanically active tissues including vocal fold tissue engineering.

In 2001, the putative genes for resilin were identified for Drosophila melanogaster and for a mosquito analogue Anopheles gambiae.[165,176,177] The successful generation of multiple recombinant versions of resilin has opened up routes to the engineering of biologically active materials with desired mechanical properties (at both low and high frequencies) for tissue regeneration applications such as vocal folds.[166,178-182] In the initial attempts to engineer such hydrogels, the Kiick laboratories have adopted 12 repeats of a 15-amino-acid repetitive 
sequence with the putative motif GGRPSDSYGAPGGGN as a structural domain to impart mechanical properties, with additional incorporation of the cell-binding ligand RGDSPG,[183] the matrix metalloproteinase (MMP)-sensitive sequence GPQGIWGQ [184,185] and the heparin-binding domain KAAKRPKAAKDKQTK to induce matrix-cell interaction.[63,186,187] The resilin-like polypeptides (RLPs) are easily crosslinked to yield tunable elastic shear and Young's moduli of values comparable to those measured for vocal fold tissues $(500-5000 \mathrm{~Pa}$ at low frequency $(1-10 \mathrm{~Hz}), 200-2000$ at higher frequency $(30-150 \mathrm{~Hz})$ and $10-50 \mathrm{kPa}$ at low strain $(<15 \%)$, respectively).[18,188-190] Hydrated, crosslinked films of RLPs also show excellent extensibility (up to $300 \%$ ), efficient recovery, negligible stress relaxation and hysteresis, as well as high resilience (97\%) characterized via standard stress-strain cyclic tensile testing, suggesting their flexibility and utility to efficiently transmit mechanical forces. Encouragingly, the RLPbased hydrogels demonstrated positive cytocompatibility and supported the adhesion and proliferation of mouse NIH3T3 fibroblasts. [188,189]

To fabricate a biomimetic microenvironment of the vocal fold, it is critical not only to provide cells a mechanically appropriate 3D matrix, but also to impart tailorable cell-matrix interactions to regulate cellular response.[147,191] Towards this purpose, a family of new RLP constructs was designed, with each polypeptide equipped with a single and different biological sequence.[192] Via this straightforward approach, it is possible to independently modulate the concentrations of cell-binding, MMP-sensitive, and heparin-sequestering domains in materials of selected mechanical properties; i.e., the biological composition can be easily decoupled from the mechanical properties of RLP-based hydrogels (Figure 10), offering exciting new opportunities in the engineering of vocal fold therapeutic materials. Cytocompatible Mannich-type condensation cross-linking chemistry was employed to facilitate fast hydrogel formation. The resulting RLP-hydrogels exhibited essentially similar elastic shear and Young's moduli across various material compositions, but demonstrated drastically different cell culture outcomes derived from the identity and concentration of selected biologically active sequences presented in the hydrogels.[192] Further mechanical characterization (e.g., oscillatory shear, uniaxial stressstrain cyclic tensile, creep, stress relaxation) of these newly synthesized RLP-hydrogels indicated reversible elongation, superior elasticity, efficient energy storage, and excellent resilience, consistent with our observation of previous characterized RLPs.[189,192-194] The mild reaction 
condition supports the viability, attachment, spreading of seeded hMSCs on the surface of RGDcontaining RLP-hydrogels. The rapid gelation permits 3D encapsulation of hMSCs in these RLP matrices without compromising cell viability or material mechanical integrity over at least 21 days. Preliminary characterization of inflammatory responses to the injectable RLP hydrogels indicated that the materials did not activate cultured macrophages or elicit significant inflammatory response after subcutaneous transplantation of the hydrogel in a rat model, suggesting the significant potential for supporting the biological and mechanical properties necessary for vocal fold in vivo application.[192-194]

In addition, the Kiick laboratories have also discovered that incorporating various RLP constructs into bulk matrices with different PEG molecules results in a liquid-liquid phase separation that positively impacts cellular behavior. Micro-scale phase separated solutions can be crosslinked into solid hydrogels via multiple crosslinking strategies, providing a versatile platform for tailoring chemistries, material composition, bulk mechanics, microstructures and scaffold degradation.[181,195-197] These systems will be advantageous for engineering growth factor sequestration and release, via the incorporation of glycosaminoglycans that will not negatively impact their elastomeric behavior, and also can be combined with in vivo cell delivery for treating VF diseases.
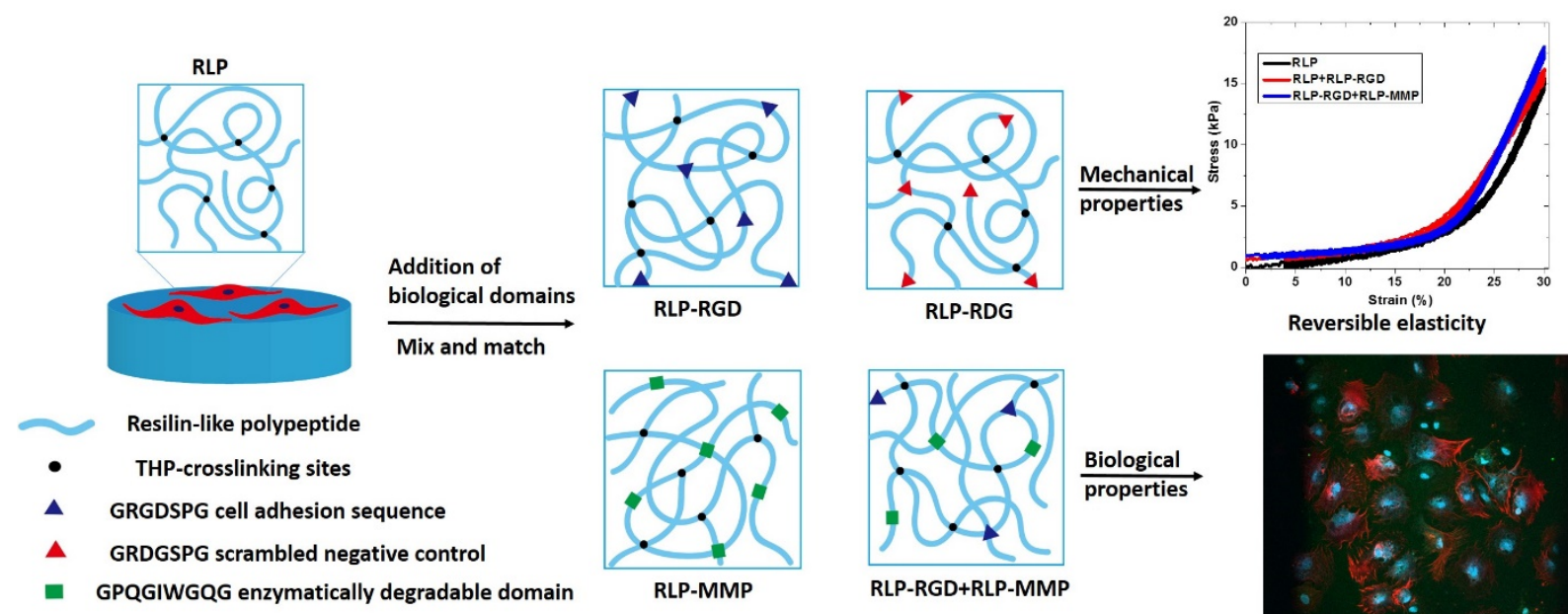

RLP-RDG

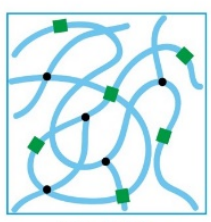

RLP-MMP

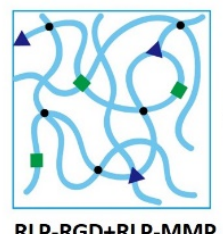

Reversible elasticity

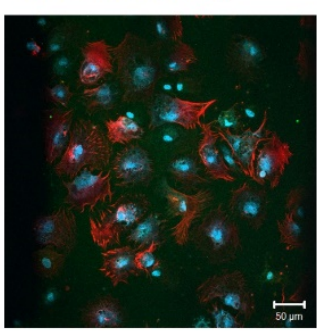

hMSCs attachment and spreading

Figure 10. Schematic of approaches for independently tailoring mechanical and biological properties of RLPs with three-repeats of stress-strain cyclic tensile testing mechanical properties at $20 \mathrm{wt} \%$ with different material compositions and 2D hMSCs attachment biological responses 
with a 20wt\% RLP-RGD hydrogel.[194] Reprint with permission from Li, L.; Mahara, A.; Tong, Z.; et al. Adv. Health. Mater. 2016, 5, 266. Copyright (2016) Wiley.

Table 1. Clinical and Experimental Biomaterials for Vocal Fold Tissue Engineering

\begin{tabular}{|c|c|c|c|c|}
\hline Biomaterial & Crosslinking method & Cell type & Animal model & $\begin{array}{c}\text { Inflammatory } \\
\text { response }\end{array}$ \\
\hline \multicolumn{5}{|c|}{$H A$ and $H A$-derivatives } \\
\hline Hylan-B (Hylaform) & Divinyl-sulfone & & Rabbit, human & Minimal inflammation \\
\hline HA-DTPH & Disulfide & $\begin{array}{l}\text { L-929 murine } \\
\text { fibroblast }\end{array}$ & Rat & Mild inflammation \\
\hline HA-DTPH+PEGDA & Michael-type addition & $\begin{array}{l}\text { T31 human tracheal } \\
\text { scar fibroblast }\end{array}$ & $\begin{array}{l}\text { Nude mice, } \\
\text { injured rabbit }\end{array}$ & Minimal inflammation \\
\hline $\begin{array}{l}\text { Carbylan-SX } \\
\text { (CMHA-S) }\end{array}$ & Michael-type addition & & Injured rabbit & Mild fibrosis \\
\hline $\begin{array}{l}\text { Carbylan-GSX } \\
\text { (Extracel) }\end{array}$ & Michael-type addition & $\begin{array}{l}\text { Immortalized hFF, } \\
\text { autologous VFF, } \\
\text { mouse bone marrow } \\
\text { MSC }\end{array}$ & $\begin{array}{l}\text { Scarred rabbit, } \\
\text { scarred rat }\end{array}$ & Mild inflammation \\
\hline \multicolumn{5}{|c|}{ Native materials and biocomposites } \\
\hline Atelogollagen & Physical interaction & Autologous MSCs & Injured canine & Minimal inflammation \\
\hline Collagen type-I & Physical interaction & $\begin{array}{l}\text { Primary VFF and } \\
\text { VFE }\end{array}$ & $\begin{array}{l}\text { Ex vivo canine } \\
\text { larynges, in vivo } \\
\text { humanized mice }\end{array}$ & Low immunogenicity \\
\hline Collagen-alginate & Physical interaction & VFF & & \\
\hline Fibrin & Physical interaction & ASC & & \\
\hline Fibrin-collagen & Physical interaction & ASC & & \\
\hline Fibrin-HA & Physical interaction & ASC & & \\
\hline HA-alginate & Physical interaction & hAdMSC & Injured rabbit & \\
\hline HA-dextran & $\begin{array}{l}\text { Hydrazide- } \\
\text { aldehyde coupling }\end{array}$ & & Ferret & Mild inflammation \\
\hline \multicolumn{5}{|l|}{ Microgels } \\
\hline HA-based & Hydrazide- & Fibroblasts & & \\
\hline
\end{tabular}




\begin{tabular}{|c|c|c|c|c|}
\hline microgels & aldehyde coupling & & & \\
\hline $\begin{array}{l}\text { HA-gelatin- } \\
\text { PEGDA }\end{array}$ & $\begin{array}{l}\text { Michael-type } \\
\text { addition }\end{array}$ & Human VFF & $\begin{array}{l}\text { Sprague- } \\
\text { Dawley rats }\end{array}$ & Mild inflammation \\
\hline \multicolumn{5}{|c|}{ Synthetic polymer materials } \\
\hline PEG30 & $\begin{array}{l}\text { Photo-initiated } \\
\text { radical } \\
\text { polymerization }\end{array}$ & Porcine VFF & Canine & $\begin{array}{l}\text { No acute } \\
\text { inflammation }\end{array}$ \\
\hline $\begin{array}{l}\text { Pluronic F127 } \\
\text { with PCL spheres }\end{array}$ & Phase separation & & Rabbit & $\begin{array}{l}\text { Minimal } \\
\text { inflammation }\end{array}$ \\
\hline $\begin{array}{l}\text { Pluronic F127 } \\
\text { with PLGA }\end{array}$ & Phase separation & & Rabbit & $\begin{array}{l}\text { Minimal } \\
\text { inflammation }\end{array}$ \\
\hline PEU & Phase separation & Cytocompatible & & \\
\hline \multicolumn{5}{|c|}{ Elastomeric Biopolymers } \\
\hline EMHP & HMDI & PVFF, NHDF & & \\
\hline $\begin{array}{l}\text { ELP4-coated } \\
\text { Tecoflex }\end{array}$ & Electrospun fibers & HVFF & & \\
\hline RLP & THP & hMSC & Mice & Mild inflammation \\
\hline
\end{tabular}

\section{Bioreactor Developments}

\subsection{Vocal fold biomechanics}

The functional agility and dexterity of vocal fold tissue during vibration are a result of the unique laminated and anisotropic structure of the vocal fold tissue. From a biomechanics perspective, vocal fold tissue must be able to sustain repetitive tension, fluid shear, and high impact collision, effectively transferring aerodynamic energy to acoustic energy for sound production.[17,198] The mechanical properties of excised vocal fold tissue have been measured under tension[199] or shear, at low and high frequencies.[18,88,190,200] Non-invasive methods have also been developed to determine the shear modulus of human vocal fold tissue in vivo by analyzing the mucosal wave propagation speed during phonation with the aid of high speed digital imaging and magnetic resonance imaging.[83]

\subsection{Bioreactor designs and high-frequency vibration outcomes}


The unique mechanical environment of vocal fold tissue suggests that cells residing in the tissue are genetically programmed to respond to vibratory forces and force gradients with high sensitivity that differentiates them from other types of fibroblasts (e.g. skin fibroblasts). Critical questions in vocal fold physiology concern the differences in composition of different layers of the LP. The mechanical environment of the cells appears to be responsible for these differences. Differences in the magnitudes of forces in the superficial and deeper layers of the lamina propria may induce expression of different genes in the different layers. These questions have motivated the development of vocal fold bioreactors to simulate the mechanical environment of the vocal fold tissue, in order to understand the effects of physiologically relevant vibrational forces on cell functions and to stimulate the cellular production of vocal fold-like ECM for vocal fold tissue repair and regeneration purposes.[201] Wolchok et al. described a simple design, in which a cell culture well was driven into oscillation at phonation frequencies by an attached electromagnetic voice coil actuator.[202] The base of the cell culture well was either a poly(dimethyl siloxane) (PDMS) sheet or a polyurethane foam, serving as a substrate or scaffold for the dynamic culture of human laryngeal fibroblasts. The study showed that vocal fold-like vibrations at $100 \mathrm{~Hz}$ at a strain up to $50 \%$ for 1-21 days influenced the expression of several key matrix and matrix related genes, enhanced the secretion of the profibrotic cytokine TGF- $\beta 1$, and increased the accumulation of the extracellular matrix proteins, such as fibronectin and type I collagen. Moreover, vibration-conditioned constructs are stiffer than the static controls.[202]

Titze and Hitchcock developed a more sophisticated bioreactor that provides both tensile and vibrational stresses.[203] In their system (Figure 11), a low frequency (or static) actuator was coupled to a series of connectors and levers to provide axial substrate elongation. The high frequency vibratory stimulus to the scaffolds was generated using a voice coil actuator connected to a lever, which is attached to a vibrational bar. This bar moves the four vibrator arms of the flask that drive the cell-encapsulated scaffolds to oscillate at frequencies of $20-200 \mathrm{~Hz}$ with strains up to $25 \%$. Using this bioreactor, Kutty et al. discovered that fibroblasts, encapsulated in a photocrosslinked hyaluronic acid hydrogel, responded to vibration by increasing the expression levels of key ECM proteins, accumulating sulfated glycosaminoglycans and decreasing collagen production. Importantly, expression levels exhibited a temporal response, with maximum 
increases observed after 3 and 5 days of vibratory stimulation and significant downregulation observed at 10 days.[204]

The Jia group (Farran et al.) used a bioreactor to investigate the effects of a range of frequencies $(60-300 \mathrm{~Hz})$ and amplitudes $(1-30 \mu \mathrm{m})$ on the behavior of neonatal foreskin fibroblasts (NFFs). They did not observe any changes in morphology of NFF growing on static versus vibrating membranes. NFF had an increase in proliferation when exposed to the $110 \mathrm{~Hz}$ frequency at $30 \mu \mathrm{m}$ amplitude, but this was not observed at any other frequency condition. They also found that lower frequency, lower amplitude conditions resulted in increased expression of collagen I. Two other ECM components involved in degradation and remodeling, tissue inhibitor of metalloproteinase 1 (TIMP) and metalloproteinase 1 (MMP1), had altered gene expression depending on frequency. Overall, this work showed the potential impact of frequency and amplitude on cellular proliferation and ECM regulation, two important variables when considering human voice production.[205]

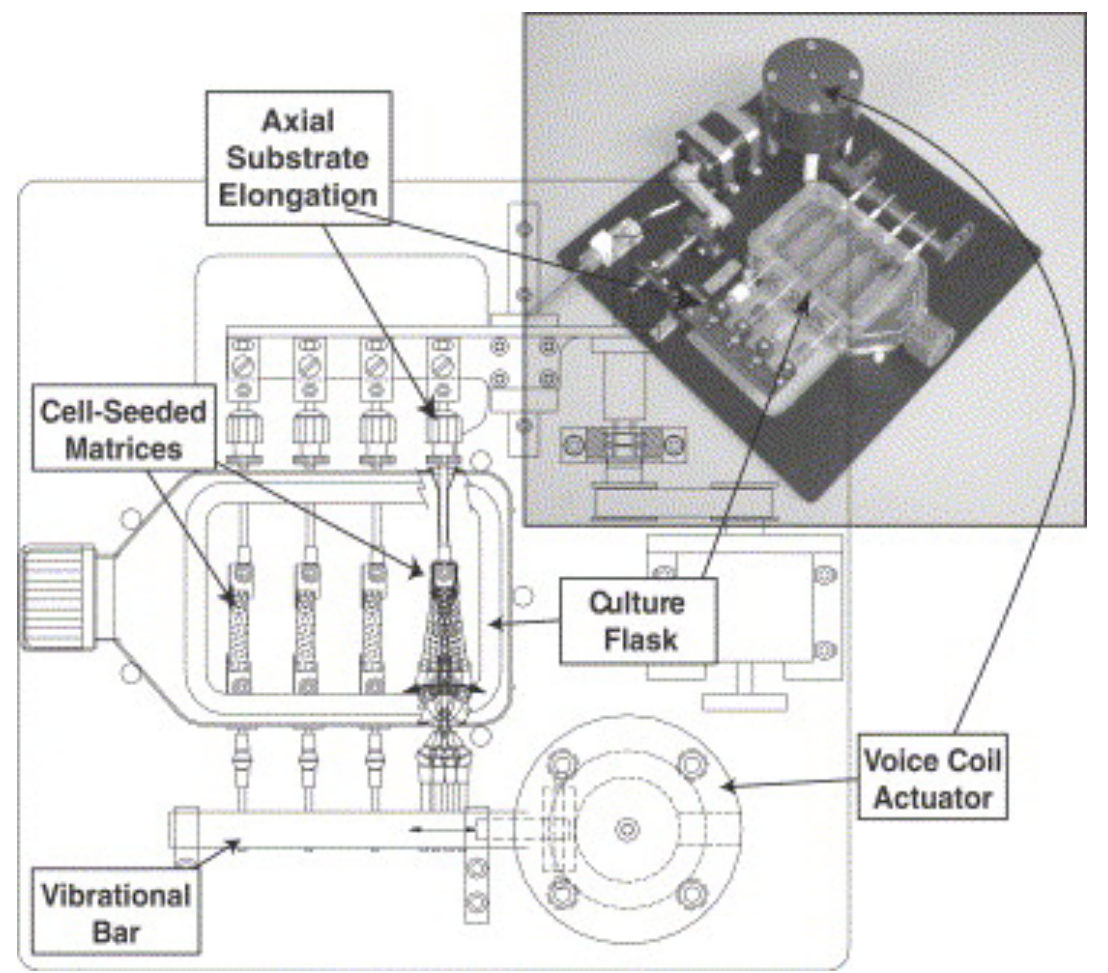

Figure 11. Schematic diagram and photograph of the Hitchcock bioreactor (named after designer), showing T-flask, substrate, and voice coil actuator.[203] Reprint with permission from Titze, I. R.; Hitchcock, R. W.; Broadhead, K.; et al. J Biomech. 2004, 37, 1521. Copyright (2004) Elsevier. 
To overcome the inertia problems associated with mechanically driven vibration devices, Tong et al. designed a bioreactor that generates vibratory stimulations by air oscillation.[201,205-207] This bioreactor is composed of two metal bars, each housing four parallel vibration modules (Figure 12). The module containing a sandwiched PDMS membrane (b(iii)) is directly mounted on top of a speaker (a(i)) that is controlled by a speaker selector. A water-tight vibration chamber is created by sandwiching an elastomeric membrane between a pair of hollow-centered acrylic blocks. The vibration signal generated by the speaker is translated to the vibration chamber by the oscillating air pressure.[201,206] Under phonation relevant conditions, the silicone membrane oscillates in an axisymmetrical fashion,[205] and the mid-membrane normal displacement $\left(\mathrm{w}_{0}\right)$ is a linear function of the driving frequency and voltage. MSC-laden fibrous polymer scaffolds were incorporated into the bioreactor and were cultured dynamically at 200 $\mathrm{Hz}$ for a total of 7 days. Cells cultured dynamically in the bioreactor were metabolically active. The reinforcement of actin filaments and the enhancement of $\alpha_{5} \beta_{1}$ integrin expression were observed under selected dynamic culture conditions. A 7-day on/off regime significantly enhanced matrix synthesis and remodeling and substantiated MSC's adaptation of the fibroblastic phenotype.[201] A follow-up investigation using the Tong bioreactor revealed that vibratory culture primed and sensitized the MSCs for the subsequent connective tissue growth factor treatment.[206] Vibrations and soluble growth factors cooperatively mediated MSCs functions, leading to an accelerated ECM synthesis and balanced ECM remodeling; and the classical Erk1/2 pathway was critically engaged in the mechano-biochemical cooperation. Limitations of the Tong bioreactor include small in-plane strain and the absence of collision forces. In addition to custom-designed devices, commercial rheometers have been adapted and modified to administer physiologically relevant shear vibrations at $20-100 \mathrm{~Hz}$ to natural or replacement tissues, while simultaneously measuring tissue viscoelastic properties.[208,209] 


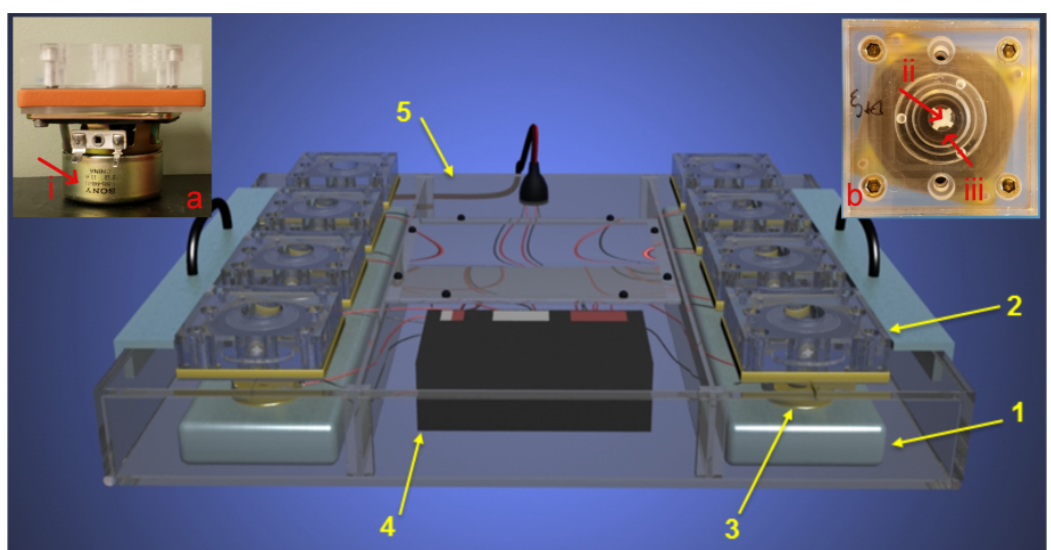

Figure 12. A custom-designed vocal fold bioreactor. (1): stationary metal bar; (2): vibration module; (3): speaker; (4): speaker selector; (5): acrylic anti-humidity chamber. Inserts: a side (a) and a top (b) view of an individual vibration module, (i): speaker; (ii): PCL mat (white); (iii): silicone elastomer (transparent) that serves the bottom of the chamber.[206] Reprint with permission from Tong, Z.; Zerdoum, A. B.; Duncan, R. L.; et al. Tissue Eng. Part A. 2014, 20, 1922. Copyright (2014) Mary Ann Liebert, Inc.

In contrast, a study by Gaston et al. examined the impact of vibration on gene expression using a bioreactor that simulates the VF by subjecting cell-seeded SECM with vibration, tensile stress and dynamic angle change three stimuli and did not find significant differences in expression of selected ECM components (collagen I, TGF- $\beta 1$, or fibronectin) between the hVFF and bone marrow-derived MSC exposed to 8 hours of vibration versus static controls.[210] However, it is possible that other ECM components, as described in Zerdoum[207] and Tong[206], may have been affected. Bartlett et al. also employed a bioreactor as a way to investigate the responses of bone marrow derived versus adipose tissue MSCs (BM-MSC vs. AT-MSC) as compared to vocal fold fibroblasts in terms of multiple strain and vibration conditions using microarray assay. Cells in the vibratory condition underwent simultaneous longitudinal tensile strain with vibrations at $200 \mathrm{~Hz}$ over a 24 hour period. No significant differences in cell proliferation were observed across various cell types or vibrational conditions. Overall, BM-MSC had more similar profiles to VFF than AT-MSC for genes involved in wound-healing based on the microarray analysis. They found lower expression of osteogenic genes, in BM-MSCs in the vibratory and stationary scaffolds as compared to cells grown on polystyrene surfaces, suggesting that the use of these scaffolds may reduce the risk of osteogenesis in this cell type.[211] The continued use of 
bioreactors as a way to investigate cellular response is necessary to evaluate potential sources for tissue regeneration in vocal fold scar.

\subsection{Current limitations and needs for advanced designs}

Although the existing vocal fold bioreactors capture certain features of the vibrating vocal fold, none fully integrates the complex biomechanical environment of the native tissue. Further, the desire to achieve mechanical stimulation at human phonation frequencies inevitably results in the introduction of undesirable physical perturbations and experimental constraints for in vitro cell culture. For example, the turbulent media flow and the inability to accommodate multiple samples limit the usage of Wolchok bioreactor in tissue engineering applications. Limitations of the Hitchcock bioreactor, on the other hand, include non-uniform strain distribution along the porous substrate,[212] the need for a large number of connectors and bars to transmit forces and the possibility of individual components to resonate at various frequencies. Finally, because the Tong bioreactor relies on the anchored silicone elastomer to transmit the oscillatory air pressure to the cellular constructs, the vibration is acoustic rather than aerodynamic, the vibration amplitude is small, and the collision forces are absent. For the rheometer-based bioreactor, although the dual capability is attractive, maintaining long-term cell viability in such a bioreactor is a significant challenge. Future effort should be dedicated to the construction and validation of a bioreactor that uses airflow to drive the synthetic vocal folds into a self-sustaining oscillatory motion with lateral collision, at the same time maintaining long term cell viability and enabling high throughput sample testing.

\section{Current Therapeutic Challenges and Future Direction}

The unique vibrational mechanical properties of human vocal folds render the delicate tissue susceptible to significant detrimental impacts that can potentially lead to voice impairment and scar formation. The establishment of the tissue engineering principle and the advancement of biotechnologies in the last decade have drastically enhanced our understanding of physiological and pathological aspects of both healthy and injured vocal fold tissues. Motivated by regenerative medicine strategies, numerous efforts have been attempted to repair and restore the functional outcomes of injured vocal folds. Encouraging advances have achieved in creating state-of-the-art scaffolds with various techniques ranging from injectable hydrogels, electrospun 
fibers, and synthetic polymers to stem cell and growth factor administration. However, the artificial biomaterials investigated so far have not recapitulated the highly complex multilayer architecture or the aerodynamic-to-acoustic energy transfer and high-frequency vibration of native lamina propria to completely restore scarred or atrophied VF. To date, most studies have focused on isolated application of an individual therapeutic methodology; further investigation should integrate degradable scaffolds, transplantation of universal cell types, controlled delivery of soluble growth factors, and dynamic mechanical stimulation into multifunctional approaches that are likely to achieve synergistic efficacy. Furthermore, it is necessary to develop continued understanding of the underlying biology of chronic vocal fold scar formation, a process that is highly relevant to multiple vocal pathologies developed progressively in response to chronic conditions. Modular biomaterials, equipped with appropriate chemical composition, hierarchical structural characteristics, physical-chemical signaling cues, and biomechanical stimuli that mimic native VF tissue have the potential to elicit encapsulated cell remodeling and induce tissue-specific ECM production that may approach scarless wound healing. The growing body of literature in vocal fold physiology and developmental biology will inspire the creation of more complex, fully functional synthetic matrices that allow the optimization of scaffold-cell interaction, correlation of the relationship between VF protein constituents and rheological outcome, as well as standardization of in vitro protocols and in vivo vocal fold animal models to generate safe and clinically useful biomaterials for patients with voice disorders.

\section{Acknowledgments}

Related work in the authors' laboratories has been supported by the National Science Foundation (DMR 0239744), the NSF Integrative Graduate and Research Traineeship (IGERT), the University of Delaware Research Foundation, the National Center for Research Resources, a component of the National Institutes of Health (P20-RR017716 and P20-RR015588), and the National Institute on Deafness and Other Communication Disorders (NIDCD RO1 DC4336, R01DC013508, RO1 DC011377A and RO1 DC014461). Its contents are solely the responsibility of the authors and do not necessarily represent the official views of NCRR or NIH.

\section{References}

[1] J. Jiang, E. Lin, D.G. Hanson, Vocal fold physiology, Otolaryngol. Clin. North Am. 33 (2000) 699-718. 
[2] S.D. Gray, F. Alipour, I.R. Titze, T.H. Hammond, Biomechanical and histologic observations of vocal fold fibrous proteins, Ann. Otol. Rhinol. Laryngol. 109 (2000) 7785.

[3] K. Sato, M. Hirano, T. Nakashima, Fine Structure of the Human Newborn and Infant Vocal Fold Mucosae, Ann. Otol. Rhinol. Laryngol. 110 (2001) 417-424.

[4] S.D. Gray, Cellular physiology of the vocal folds, Otolaryngol Clin North Am. 33 (2000) 679-698.

[5] M.S. Benninger, D. Alessi, S. Archer, R. Bastian, C. Ford, J. Koufman, et al., Vocal fold scarring: Current concepts and management, Otolaryngol. Neck Surg. 115 (1996) 474482.

[6] L.O. Ramig, K. Verdolini, Treatment efficacy voice disorders, J. Speech, Lang. Hear. Res. 41 (1998) S101-S116.

[7] J.A. Koufman, P.D. Blalock, Functional voice disorders, Otolaryngol. Clin. North Am. 24 (1991) 1059-1073.

[8] J.K. Hansen, S.L. Thibeault, Current understanding and review of the literature: vocal fold scarring., J. Voice. 20 (2006) 110-120.

[9] J.K. Kutty, K. Webb, Tissue Engineering Therapies for the Vocal Fold Lamina Propria, Tissue Eng. Part B Rev. 15 (2009) 249-262.

[10] R.S. Bartlett, S.L. Thibeault, G.D. Prestwich, Therapeutic potential of gel-based injectables for vocal fold regeneration, Biomed. Mater. 7 (2012) 24103.

[11] C.M. Sapienza, B.H. Ruddy, Voice Disorders, Plural Pub., 2013.

[12] N. Roy, R.M. Merrill, S.D. Gray, E.M. Smith, Voice Disorders in the General Population: Prevalence, Risk Factors, and Occupational Impact, Laryngoscope. 115 (2005) 19881995.

[13] B. Fritzell, Voice disorders and occupations, Logop. Phoniatr. Vocology. 21 (1996) 7-12.

[14] S.M. Zeitels, R.E. Hillman, M. Mauri, R. Desloge, P.B. Doyle, Phonomicrosurgery in singers and performing artists: Treatment outcomes, management theories, and future directions, Ann. Otol. Rhinol. Laryngol. 111 (2002) 21-40.

[15] K. Verdolini, L.O. Ramig, Review: Occupational risks for voice problems, Logop. Phoniatr. Vocology. 26 (2001) 37-46.

[16] D.E. Rosow, M. Szczupak, S. Saint-Victor, J.D. Gerhard, C. DuPont, K. Lo, The economic impact of vocal attrition in public school teachers in Miami-Dade County, Laryngoscope. 126 (2016) 665-671.

[17] I.R. Titze, Principles of Voice Production, Prentice Hall, 1994.

[18] S.S. Teller, A.J.E. Farran, L. Xiao, T. Jiao, R.L. Duncan, R.J. Clifton, et al., HighFrequency Viscoelastic Shear Properties of Vocal Fold Tissues: Implications for Vocal Fold Tissue Engineering, Tissue Eng. Part A. 18 (2012) 2008-2019.

[19] A.K. Miri, Mechanical Characterization of Vocal Fold Tissue: A Review Study., J. Voice. 
28 (2014) 657-667.

[20] T. Haji, K. Mori, K. Omori, N. Isshiki, Mechanical Properties of the Vocal Fold, Acta Otolaryngol. 112 (1992) 559-565.

[21] C. Leydon, R.S. Bartlett, D.A. Roenneburg, S.L. Thibeault, Localization of LabelRetaining Cells in Murine Vocal Fold Epithelium, J. Speech, Lang. Hear. Res. 54 (2011) 1060-1066.

[22] J. Moore, S. Thibeault, Insights into the role of elastin in vocal fold health and disease., J. Voice. 26 (2012) 269-275.

[23] K. Ishii, W.G. Zhai, M. Akita, H. Hirose, Ultrastructure of the Lamina Propria of the Human Vocal Fold, Acta Otolaryngol. 116 (1996) 778-782.

[24] E.C. de Melo, M. Lemos, J. Aragão Ximenes Filho, L.U. Sennes, P.H. Nascimento Saldiva, D.H. Tsuji, Distribution of Collagen in the Lamina Propria of the Human Vocal Fold, Laryngoscope. 113 (2003) 2187-2191.

[25] S.L. Thibeault, S.D. Gray, D.M. Bless, R.W. Chan, C.N. Ford, Histologic and Rheologic Characterization of Vocal Fold Scarring, J. Voice. 16 (2002) 96-104.

[26] R.T. Sataloff, Sataloff's Comprehensive Textbook of Otolaryngology: Head \& Neck Surgery: Six Volume Set, Jaypee Brothers,Medical Publishers Pvt. Limited, 2015.

[27] M. Graupp, S. Bachna-Rotter, C. Gerstenberger, G. Friedrich, E. Fröhlich-Sorger, K. Kiesler, et al., The unsolved chapter of vocal fold scars and how tissue engineering could help us solve the problem, Eur. Arch. Oto-Rhino-Laryngology. (2015) 1-6.

[28] E.E. Levendoski, C. Leydon, S.L. Thibeault, Vocal Fold Epithelial Barrier in Health and Injury: A Research Review, J. Speech, Lang. Hear. Res. 57 (2014) 1679-1691.

[29] J.R. Dowdall, P.M. Sadow, C. Hartnick, V. Vinarsky, H. Mou, R. Zhao, et al., Identification of distinct layers within the stratified squamous epithelium of the adult human true vocal fold, Laryngoscope. 125 (2015) E313-E319.

[30] M. Hirano, Morphological Structure of the Vocal Cord as a Vibrator and its Variations, Folia Phoniatr. Logop. 26 (1974) 89-94.

[31] I.R. Titze, J. Jiang, D.G. Drucker, Preliminaries to the body-cover theory of pitch control, J. Voice. 1 (1988) 314-319.

[32] I.R. Titze, Phonation threshold pressure: A missing link in glottal aerodynamics, J. Acoust. Soc. Am. 91 (1992) 2926-2935.

[33] I.R. Titze, The physics of small-amplitude oscillation of the vocal folds, J. Acoust. Soc. Am. 83 (1988) 1536-1552.

[34] J. van den Berg, On the Air Resistance and the Bernoulli Effect of the Human Larynx, J. Acoust. Soc. Am. 29 (1957) 626-631.

[35] I.R. Titze, Vocal Fold Physiology: Frontiers in Basic Science, Illustrate, Singular Publishing Group, 1993.

[36] R. Speyer, G.H. Wieneke, I. van Wijck-Warnaar, P.H. Dejonckere, Effects of voice 
therapy on the voice range profiles of dysphonic patients, J. Voice. 17 (2003) 544-556.

[37] I.R. Titze, Voice Training and Therapy With a Semi-Occluded Vocal Tract: Rationale and Scientific Underpinnings, J. Speech, Lang. Hear. Res. 49 (2006) 448-459.

[38] P.S. Mallur, C.A. Rosen, Vocal Fold Injection: Review of Indications, Techniques, and Materials for Augmentation, Clin. Exp. Otorhinolaryngol. 3 (2010) 177-182.

[39] S. Hirano, Current treatment of vocal fold scarring, Curr. Opin. Otolaryngol. Head Neck Surg. 13 (2005) 143-147.

[40] G. Friedrich, F.G. Dikkers, C. Arens, M. Remacle, M. Hess, A. Giovanni, et al., Vocal fold scars: current concepts and future directions. Consensus report of the phonosurgery committee of the European laryngological society, Eur. Arch. Oto-Rhino-Laryngology. 270 (2013) 2491-2507.

[41] A. Ben-Yakar, C. Hoy, W.N. Everett, J. Kobler, Toward endoscopic ultrafast laser microsurgery of vocal folds, J. Biomed. Opt. 17 (2012) 038002.

[42] N. V Welham, S.H. Choi, S.H. Dailey, C.N. Ford, J.J. Jiang, D.M. Bless, Prospective multi-arm evaluation of surgical treatments for vocal fold scar and pathologic sulcus vocalis, Laryngoscope. 121 (2011) 1252-1260.

[43] D.M. Bless, N. V Welham, Characterization of vocal fold scar formation, prophylaxis, and treatment using animal models, Curr. Opin. Otolaryngol. Head Neck Surg. 18 (2010) 481486.

[44] T. Tateya, J.H. Sohn, I. Tateya, D.M. Bless, Histologic Characterization of Rat Vocal Fold Scarring, Ann. Otol. Rhinol. Laryngol. 114 (2005) 183-191.

[45] B. Rousseau, S. Hirano, T.D. Scheidt, N. V Welham, S.L. Thibeault, R.W. Chan, et al., Characterization of Vocal Fold Scarring in a Canine Model, Laryngoscope. 113 (2003) 620-627.

[46] G. Woodson, Developing a Porcine Model for Study of Vocal Fold Scar, J. Voice. 26 (2012) 706-710.

[47] S. Hirano, Y. Kishimoto, A. Suehiro, S. Kanemaru, J. Ito, Regeneration of aged vocal fold: First human case treated with fibroblast growth factor, Laryngoscope. 119 (2009) 197-202.

[48] T. Ohno, M.J. Yoo, E.R. Swanson, S. Hirano, R.H. Ossoff, B. Rousseau, Regenerative effects of basic fibroblast growth factor on extracellular matrix production in aged rat vocal folds, Ann. Otol. Rhinol. Laryngol. 118 (2009) 559-564.

[49] T. Kanazawa, D. Komazawa, K. Indo, Y. Akagi, Y. Lee, K. Nakamura, et al., Single injection of basic fibroblast growth factor to treat severe vocal fold lesions and vocal fold paralysis, Laryngoscope. 125 (2015) E338-E344.

[50] D.M. Bless, N. V Welham, S. Hirano, H. Nagai, D.W. Montequin, B. Rousseau, et al., Growth Factor Therapy for Vocal Fold Scarring in a Canine Model, Ann. Otol. Rhinol. Laryngol. 113 (2004) 777-785.

[51] T. Ohno, S. Hirano, S.-I. Kanemaru, M. Yamashita, H. Umeda, A. Suehiro, et al., Drug 
Delivery System of Hepatocyte Growth Factor for the Treatment of Vocal Fold Scarring in a Canine Model, Ann. Otol. Rhinol. Laryngol. 116 (2007) 762-769.

[52] Y. Kishimoto, S. Hirano, Y. Kitani, A. Suehiro, H. Umeda, I. Tateya, et al., Chronic vocal fold scar restoration with hepatocyte growth factor hydrogel, Laryngoscope. 120 (2010) $108-113$.

[53] M. Gugatschka, S. Ohno, A. Saxena, S. Hirano, Regenerative medicine of the larynx. Where are we today? A review., J. Voice. 26 (2012) 670.e7-13.

[54] J.J. Rice, M.M. Martino, L. De Laporte, F. Tortelli, P.S. Briquez, J.A. Hubbell, Engineering the Regenerative Microenvironment with Biomaterials, Adv. Healthc. Mater. 2 (2013) 57-71.

[55] L.G. Griffith, G. Naughton, Tissue Engineering--Current Challenges and Expanding Opportunities, Science. 295 (2002) 1009-1014.

[56] R. Langer, D.A. Tirrell, Designing materials for biology and medicine, Nature. 428 (2004) 487-492.

[57] M.P. Lutolf, J.A. Hubbell, Synthetic biomaterials as instructive extracellular microenvironments for morphogenesis in tissue engineering, Nat. Biotechnol. 23 (2005) 47-55.

[58] K. Ghosh, D.E. Ingber, Micromechanical control of cell and tissue development: implications for tissue engineering., Adv. Drug Deliv. Rev. 59 (2007) 1306-18.

[59] J.A. Hubbell, R. Langer, Translating materials design to the clinic, Nat Mater. 12 (2013) 963-966.

[60] K. Lee, J.A. Hubbell, Tissue, cell and engineering., Curr. Opin. Biotechnol. 24 (2013) $827-829$.

[61] M.M. Martino, P.S. Briquez, E. Güç, F. Tortelli, W.W. Kilarski, S. Metzger, et al., Growth Factors Engineered for Super-Affinity to the Extracellular Matrix Enhance Tissue Healing, Science. 343 (2014) 885-888.

[62] S.A. Maskarinec, D.A. Tirrell, Protein engineering approaches to biomaterials design, Curr. Opin. Biotechnol. 16 (2005) 422-426.

[63] K.L. Kiick, Peptide- and protein-mediated assembly of heparinized hydrogels, Soft Matter. 4 (2008) 29-37.

[64] D.E. Discher, D.J. Mooney, P.W. Zandstra, Growth Factors, Matrices, and Forces Combine and Control Stem Cells, Science 80. 324 (2009) 1673-1677.

[65] J. Gaston, S.L. Thibeault, Hyaluronic acid hydrogels for vocal fold wound healing, Biomatter. 3 (2013) e23799.

[66] J.A. Burdick, G.D. Prestwich, Hyaluronic Acid Hydrogels for Biomedical Applications, Adv. Mater. 23 (2011) H41-H56.

[67] M.S. Hahn, J.B. Kobler, B.C. Starcher, S.M. Zeitels, R. Langer, Quantitative and Comparative Studies of the Vocal Fold Extracellular Matrix I: Elastic Fibers and 
Hyaluronic Acid, Ann. Otol. Rhinol. Laryngol. 115 (2006) 156-164.

[68] R.W. Chan, S.D. Gray, I.R. Titze, The importance of hyaluronic acid in vocal fold biomechanics, Otolaryngol. Head Neck Surg. 124 (2001) 607-614.

[69] J.E. Butler, T.H. Hammond, S.D. Gray, Gender-Related Differences of Hyaluronic Acid Distribution in the Human Vocal Fold, Laryngoscope. 111 (2001) 907-911.

[70] J. Entwistle, C.L. Hall, E.A. Turley, HA receptors: Regulators of signalling to the cytoskeleton, J. Cell. Biochem. 61 (1996) 569-577.

[71] J.R.E. Fraser, T.C. Laurent, U.B.G. Laurent, Hyaluronan: its nature, distribution, functions and turnover, J. Intern. Med. 242 (1997) 27-33.

[72] S.L. Thibeault, S.A. Klemuk, X. Chen, B.H. Quinchia Johnson, In Vivo engineering of the vocal fold ECM with injectable HA hydrogels-late effects on tissue repair and biomechanics in a rabbit model., J. Voice. 25 (2011) 249-253.

[73] C.L. L. Hallén C. Johansson, Cross-linked Hyaluronan (Hylan B Gel): a New Injectable Remedy for Treatment of Vocal Fold Insufficiency - an Animal Study, Acta Otolaryngol. 119 (1999) 107-111.

[74] S. Hertegård, L. Hallén, C. Laurent, E. Lindström, K. Olofsson, P. Testad, et al., CrossLinked Hyaluronan Used as Augmentation Substance for Treatment of Glottal Insufficiency: Safety Aspects and Vocal Fold Function, Laryngoscope. 112 (2002) 22112219.

[75] S. Hertegård, A. Dahlqvist, C. Laurent, A. Borzacchiello, L. Ambrosio, Viscoelastic properties of rabbit vocal folds after augmentation., Otolaryngol. Head. Neck Surg. 128 (2003) 401-406.

[76] Å. Dahlqvist, O. Gärskog, C. Laurent, S. Hertegård, L. Ambrosio, A. Borzacchiello, Viscoelasticity of Rabbit Vocal Folds After Injection Augmentation, Laryngoscope. 114 (2004) 138-142.

[77] X. Jia, J.A. Burdick, J. Kobler, R.J. Clifton, J.J. Rosowski, S.M. Zeitels, et al., Synthesis and Characterization of in Situ Cross-Linkable Hyaluronic Acid-Based Hydrogels with Potential Application for Vocal Fold Regeneration, Macromolecules. 37 (2004) 32393248.

[78] X.Z. Shu, Y. Liu, Y. Luo, M.C. Roberts, G.D. Prestwich, Disulfide Cross-Linked Hyaluronan Hydrogels, Biomacromolecules. 3 (2002) 1304-1311.

[79] Y. Liu, X. Zheng Shu, G.D. Prestwich, Biocompatibility and stability of disulfidecrosslinked hyaluronan films, Biomaterials. 26 (2005) 4737-4746.

[80] X. Zheng Shu, Y. Liu, F.S. Palumbo, Y. Luo, G.D. Prestwich, In situ crosslinkable hyaluronan hydrogels for tissue engineering, Biomaterials. 25 (2004) 1339-1348.

[81] J.K. Hansen, S.L. Thibeault, J.F. Walsh, X.Z. Shu, G.D. Prestwich, In Vivo Engineering of the Vocal Fold Extracellular Matrix with Injectable Hyaluronic Acid Hydrogels: Early Effects on Tissue Repair and Biomechanics in a Rabbit Model, Ann. Otol. Rhinol. Laryngol. 114 (2005) 662-670. 
[82] H.K. Heris, A.K. Miri, U. Tripathy, F. Barthelat, L. Mongeau, Indentation of poroviscoelastic vocal fold tissue using an atomic force microscope, J. Mech. Behav. Biomed. Mater. 28 (2013) 383-392.

[83] S. Kazemirad, H. Bakhshaee, L. Mongeau, K. Kost, Non-invasive in vivo measurement of the shear modulus of human vocal fold tissue, J Biomech. 47 (2014) 1173-1179.

[84] S. Kazemirad, H.K. Heris, L. Mongeau, Viscoelasticity of hyaluronic acid-gelatin hydrogels for vocal fold tissue engineering, J. Biomed. Mater. Res. Part B Appl. Biomater. 104 (2016) 283-290.

[85] X. Chen, S.L. Thibeault, Biocompatibility of a synthetic extracellular matrix on immortalized vocal fold fibroblasts in 3-D culture., Acta Biomater. 6 (2010) 2940-2948.

[86] X. Chen, S.L. Thibeault, Role of tumor necrosis factor- $\alpha$ in wound repair in human vocal fold fibroblasts, Laryngoscope. 120 (2010) 1819-1825.

[87] S. Duflo, S.L. Thibeault, W. Li, X.Z. Shu, G.D. Prestwich, Vocal fold tissue repair in vivo using a synthetic extracellular matrix, Tissue Eng. 12 (2006) 2171-2180.

[88] R.W. Chan, I.R. Titze, Viscoelastic shear properties of human vocal fold mucosa: Measurement methodology and empirical results, J. Acoust. Soc. Am. 106 (1999) 20082021.

[89] S. Duflo, S.L. Thibeault, W. Li, X.Z. Shu, G. Prestwich, Effect of a synthetic extracellular matrix on vocal fold lamina propria gene expression in early wound healing, Tissue Eng. 12 (2006) 3201-3207.

[90] S.L. Thibeault, S.A. Klemuk, M.E. Smith, C. Leugers, G. Prestwich, In Vivo Comparison of Biomimetic Approaches for Tissue Regeneration of the Scarred Vocal Fold, Tissue Eng. Part A. 15 (2008) 1481-1487.

[91] B. Quinchia Johnson, R. Fox, X. Chen, S. Thibeault, Tissue regeneration of the vocal fold using bone marrow mesenchymal stem cells and synthetic extracellular matrix injections in rats, Laryngoscope. 120 (2010) 537-545.

[92] J.A. Hubbell, Biomaterials in tissue engineering, Bio-Technology. 13 (1995) 565-576.

[93] S.-I. Kanemaru, H. Kojima, S. Hirano, T. Nakamura, A. Magrufov, J. Ito, et al., Regeneration of the Vocal Fold Using Autologous Mesenchymal Stem Cells, Ann. Otol. Rhinol. Laryngol. 112 (2003) 915-920.

[94] Y. Kishirnoto, S. Hirano, T. Kojima, S. Kanemaru, J. Ito, Implantation of an Atelocollagen Sheet for the Treatment of Vocal Fold Scarring and Sulcus Vocalis, Ann. Otol. Rhinol. Laryngol. 118 (2009) 613-620.

[95] Y. Kishimoto, N. V Welham, S. Hirano, Implantation of atelocollagen sheet for vocal fold scar, Curr. Opin. Otolaryngol. Head Neck Surg. 18 (2010) 507-511.

[96] J.L. Long, P. Zuk, G.S. Berke, D.K. Chhetri, Epithelial differentiation of adipose-derived stem cells for laryngeal tissue engineering, Laryngoscope. 120 (2010) 125-131.

[97] J.L. Long, J. Neubauer, Z. Zhang, P. Zuk, G.S. Berke, D.K. Chhetri, Functional testing of a tissue-engineered vocal fold cover replacement, Otolaryngol.- Head Neck Surg. 142 
(2010) 438-440.

[98] T. Caton, S.L. Thibeault, S. Klemuk, M.E. Smith, Viscoelasticity of Hyaluronan and Nonhyaluronan Based Vocal Fold Injectables: Implications for Mucosal Versus Muscle Use, Laryngoscope. 117 (2007) 516-521.

[99] C.N. Ford, D.M. Bless, Collagen injection in the scarred vocal fold, J. Voice. 1 (1987) $116-118$.

[100] C. Ling, Q. Li, M.E. Brown, Y. Kishimoto, Y. Toya, E.E. Devine, et al., Bioengineered vocal fold mucosa for voice restoration, Sci. Transl. Med. 7 (2015) 314ra187.

[101] X. Hu, P. Cebe, A.S. Weiss, F. Omenetto, D.L. Kaplan, Protein-based composite materials, Mater. Today. 15 (2012) 208-215.

[102] H.K. Lau, K.L. Kiick, Opportunities for Multicomponent Hybrid Hydrogels in Biomedical Applications, Biomacromolecules. 16 (2015) 28-42.

[103] R. Rajkhowa, E.S. Gil, J. Kluge, K. Numata, L. Wang, X. Wang, et al., Reinforcing silk scaffolds with silk particles., Macromol. Biosci. 10 (2010) 599-611.

[104] M.S. Hahn, B.A. Teply, M.M. Stevens, S.M. Zeitels, R. Langer, Collagen composite hydrogels for vocal fold lamina propria restoration., Biomaterials. 27 (2006) 1104-1109.

[105] J.M. Caves, V.A. Kumar, A.W. Martinez, J. Kim, C.M. Ripberger, C.A. Haller, et al., The use of microfiber composites of elastin-like protein matrix reinforced with synthetic collagen in the design of vascular grafts., Biomaterials. 31 (2010) 7175-7182.

[106] G.K. Qin, S. Lapidot, K. Numata, X. Hu, S. Meirovitch, M. Dekel, et al., Expression, Cross-Linking, and Characterization of Recombinant Chitin Binding Resilin, Biomacromolecules. 10 (2009) 3227-3234.

[107] G.C. Yeo, B. Aghaei-Ghareh-Bolagh, E.P. Brackenreg, M.A. Hiob, P. Lee, A.S. Weiss, Fabricated Elastin, Adv. Healthc. Mater. 4 (2015) 2530-2556.

[108] T. Huang, H.G. Xu, K.X. Jiao, L.P. Zhu, H.R. Brown, H.L. Wang, A Novel Hydrogel with High Mechanical Strength: A Macromolecular Microsphere Composite Hydrogel, Adv. Mater. 19 (2007) 1622-1626.

[109] H. Park, S. Karajanagi, K. Wolak, J. Aanestad, L. Daheron, J.B. Kobler, et al., ThreeDimensional Hydrogel Model Using Adipose-Derived Stem Cells for Vocal Fold Augmentation, Tissue Eng. Part A. 16 (2009) 535-543.

[110] S.L. Rowe, J.P. Stegemann, Interpenetrating Collagen-Fibrin Composite Matrices with Varying Protein Contents and Ratios, Biomacromolecules. 7 (2006) 2942-2948.

[111] S.Y. Na, S.H. Oh, K.S. Song, J.H. Lee, Hyaluronic acid/mildly crosslinked alginate hydrogel as an injectable tissue adhesion barrier, J. Mater. Sci. Med. 23 (2012) 23032313.

[112] Y.-M. Kim, S.H. Oh, J.-S. Choi, S. Lee, J.C. Ra, J.H. Lee, et al., Adipose-derived stem cell-containing hyaluronic acid/alginate hydrogel improves vocal fold wound healing, Laryngoscope. 124 (2014) E64-E72. 
[113] Y. Luo, J.B. Kobler, J.T. Heaton, X. Jia, S.M. Zeitels, R. Langer, Injectable hyaluronic acid-dextran hydrogels and effects of implantation in ferret vocal fold., J. Biomed. Mater. Res. B. Appl. Biomater. 93 (2010) 386-393.

[114] J.R. Tse, J.L. Long, Microstructure characterization of a decellularized vocal fold scaffold for laryngeal tissue engineering, Laryngoscope. 124 (2014) E326-E331.

[115] Q. Li, Z. Chang, G. Oliveira, M. Xiong, L.M. Smith, B.L. Frey, et al., Protein turnover during in vitro tissue engineering, Biomaterials. 81 (2016) 104-113.

[116] E.A. Wrona, R. Peng, H. Born, M.R. Amin, R.C. Branski, D.O. Freytes, Derivation and characterization of porcine vocal fold extracellular matrix scaffold, Laryngoscope. 126 (2016) 928-935.

[117] A. Khademhosseini, R. Langer, Microengineered hydrogels for tissue engineering, Biomaterials. 28 (2007) 5087-5092.

[118] J.K. Oh, R. Drumright, D.J. Siegwart, K. Matyjaszewski, The development of microgels/nanogels for drug delivery applications, Prog. Polym. Sci. 33 (2008) 448-477.

[119] W. Zhou, J.M. Stukel, H.L. Cebull, R.K. Willits, Tuning the Mechanical Properties of Poly(Ethylene Glycol) Microgel-Based Scaffolds to Increase 3D Schwann Cell Proliferation, Macromol. Biosci. 16 (2016) 535-544.

[120] X. Jia, Y. Yeo, R.J. Clifton, T. Jiao, D.S. Kohane, J.B. Kobler, et al., Hyaluronic AcidBased Microgels and Microgel Networks for Vocal Fold Regeneration, Biomacromolecules. 7 (2006) 3336-3344.

[121] N. Sahiner, A.K. Jha, D. Nguyen, X. Jia, Fabrication and characterization of crosslinkable hydrogel particles based on hyaluronic acid: potential application in vocal fold regeneration, J. Biomater. Sci. Polym. Ed. 19 (2008) 223-243.

[122] A.K. Jha, R.A. Hule, T. Jiao, S.S. Teller, R.J. Clifton, R.L. Duncan, et al., Structural Analysis and Mechanical Characterization of Hyaluronic Acid-Based Doubly CrossLinked Networks, Macromolecules. 42 (2009) 537-546.

[123] H.K. Heris, M. Rahmat, L. Mongeau, Characterization of a Hierarchical Network of Hyaluronic Acid/Gelatin Composite for use as a Smart Injectable Biomaterial, Macromol. Biosci. 12 (2012) 202-210.

[124] H.K. Heris, J. Daoud, S. Sheibani, H. Vali, M. Tabrizian, L. Mongeau, Investigation of the Viability, Adhesion, and Migration of Human Fibroblasts in a Hyaluronic Acid/Gelatin Microgel-Reinforced Composite Hydrogel for Vocal Fold Tissue Regeneration, Adv. Healthc. Mater. 5 (2016) 255-265.

[125] J.M.S. Coppoolse, T.G. Van Kooten, H.K. Heris, L. Mongeau, N.Y.K. Li, S.L. Thibeault, et al., An In Vivo Study of Composite Microgels Based on Hyaluronic Acid and Gelatin for the Reconstruction of Surgically Injured Rat Vocal Folds, J. Speech, Lang. Hear. Res. 57 (2014) S658-S673.

[126] N. Isshiki, K. Shoji, H. Kojima, S. Hirano, Vocal Fold Atrophy and its Surgical Treatment, Ann. Otol. Rhinol. Laryngol. 105 (1996) 182-188. 
[127] S.H. Dailey, C.N. Ford, Surgical Management of Sulcus Vocalis and Vocal Fold Scarring, Otolaryngol. Clin. North Am. 39 (2006) 23-42.

[128] E.S. Place, N.D. Evans, M.M. Stevens, Complexity in biomaterials for tissue engineering, Nat. Mater. 8 (2009) 457-470.

[129] C.A. DeForest, K.S. Anseth, Advances in Bioactive Hydrogels to Probe and Direct Cell Fate, Annu. Rev. Chem. Biomol. Eng. 3 (2012) 421-444.

[130] A.M. Rosales, K.S. Anseth, The design of reversible hydrogels to capture extracellular matrix dynamics, Nat. Rev. Mater. 1 (2016) 15012.

[131] H. Liao, D. Munoz-Pinto, X. Qu, Y. Hou, M.A. Grunlan, M.S. Hahn, Influence of hydrogel mechanical properties and mesh size on vocal fold fibroblast extracellular matrix production and phenotype, Acta Biomater. 4 (2008) 1161-1171.

[132] S.S. Karajanagi, G. Lopez-Guerra, H. Park, J.B. Kobler, M. Galindo, J. Aanestad, et al., Assessment of Canine Vocal Fold Function after Injection of a New Biomaterial Designed to Treat Phonatory Mucosal Scarring, Ann. Otol. Rhinol. Laryngol. 120 (2011) 175-184.

[133] S.K. Kwon, J.-J. Song, C.G. Cho, S.-W. Park, S.J. Choi, S.H. Oh, et al., Polycaprolactone spheres and theromosensitive pluronic F127 hydrogel for vocal fold augmentation: In vivo animal study for the treatment of unilateral vocal fold palsy, Laryngoscope. 123 (2013) 1694-1703.

[134] J.H. Lee, D.W. Kim, E.N. Kim, S.-W. Park, H.-B. Kim, S.H. Oh, et al., Evaluation of the Poly(lactic-co-glycolic acid)/Pluronic F127 for Injection Laryngoplasty in Rabbits, Otolaryngol. - Head Neck Surg. 151 (2014) 830-835.

[135] J. Gaston, R.S. Bartlett, S.A. Klemuk, S.L. Thibeault, Formulation and Characterization of a Porous, Elastomeric Biomaterial for Vocal Fold Tissue Engineering Research, Ann. Otol. Rhinol. Laryngol. 123 (2014) 866-874.

[136] T.H. Hammond, R. Zhou, E.H. Hammond, A. Pawlak, S.D. Gray, The intermediate layer: A morphologic study of the elastin and hyaluronic acid constituents of normal human vocal folds, J. Voice. 11 (1997) 59-66.

[137] T.H. Hammond, S.D. Gray, J. Butler, R.X. Zhou, E. Hammond, Age- and gender-related elastin distribution changes in human vocal folds, Otolaryngol. Neck Surg. 119 (1998) 314-322.

[138] B.T. Steven D. Gray Karen J. Chan, Dissection Plane of the Human Vocal Fold Lamina Propria and Elastin Fibre Concentration, Acta Otolaryngol. 120 (2000) 87-91.

[139] G.W.G. Chalmers, J.M. Gosline, M.A. Lillie, The hydrophobicity of vertebrate elastins, J. Exp. Biol. 202 (1999) 301-314.

[140] B. Vrhovski, A.S. Weiss, Biochemistry of tropoelastin, Eur. J. Biochem. 258 (1998) 1-18.

[141] W.F. Daamen, J.H. Veerkamp, J.C.M. van Hest, T.H. van Kuppevelt, Elastin as a biomaterial for tissue engineering, Biomaterials. 28 (2007) 4378-4398.

[142] J. Gosline, M. Lillie, E. Carrington, P. Guerette, C. Ortlepp, K. Savage, Elastic proteins: biological roles and mechanical properties, Philos. Trans. R. Soc. London Ser. B- 
Biological Sci. 357 (2002) 121-132.

[143] M.B. Van Eldijk, C.L. McGann, K.L. Kiick, J.M. Van Hest, Elastomeric Polypeptides, in: Pept. Mater., Springer Berlin Heidelberg, 2012: pp. 71-116.

[144] D.L. Nettles, A. Chilkoti, L.A. Setton, Applications of elastin-like polypeptides in tissue engineering., Adv. Drug Deliv. Rev. 62 (2010) 1479-1485.

[145] L. Li, M.B. Charati, K.L. Kiick, Elastomeric polypeptide-based biomaterials, Polym. Chem. 1 (2010) 1160-1170.

[146] J.E. Gagner, W. Kim, E.L. Chaikof, Designing protein-based biomaterials for medical applications, Acta Biomater. 10 (2014) 1542-1557.

[147] D. Sengupta, S.C. Heilshorn, Protein-Engineered Biomaterials: Highly Tunable Tissue Engineering Scaffolds, Tissue Eng. Part B-Reviews. 16 (2009) 285-293.

[148] C. Hrabchak, J. Rouleau, I. Moss, K. Woodhouse, M. Akens, C. Bellingham, et al., Assessment of biocompatibility and initial evaluation of genipin cross-linked elastin-like polypeptides in the treatment of an osteochondral knee defect in rabbits., Acta Biomater. 6 (2010) 2108-2115.

[149] D.W. Urry, T. Hugel, M. Seitz, H.E. Gaub, L. Sheiba, J. Dea, et al., Elastin: a representative ideal protein elastomer, Philos. Trans. R. Soc. London Ser. B-Biological Sci. 357 (2002) 169-184.

[150] S.E. Grieshaber, A.J.E. Farran, S. Lin-Gibson, K.L. Kiick, X. Jia, Synthesis and Characterization of Elastin-Mimetic Hybrid Polymers with Multiblock, Alternating Molecular Architecture and Elastomeric Properties, Macromolecules. 42 (2009) 25322541.

[151] C.A. DeForest, B.D. Polizzotti, K.S. Anseth, Sequential click reactions for synthesizing and patterning three-dimensional cell microenvironments, Nat Mater. 8 (2009) 659-664.

[152] C.A. DeForest, K.S. Anseth, Cytocompatible click-based hydrogels with dynamically tunable properties through orthogonal photoconjugation and photocleavage reactions, Nat Chem. 3 (2011) 925-931.

[153] S.E. Grieshaber, A.J.E. Farran, S. Bai, K.L. Kiick, X. Jia, Tuning the Properties of Elastin Mimetic Hybrid Copolymers via a Modular Polymerization Method, Biomacromolecules. 13 (2012) 1774-1786.

[154] L.A. Hughes, J. Gaston, K. McAlindon, K.A. Woodhouse, S.L. Thibeault, Electrospun fiber constructs for vocal fold tissue engineering: effects of alignment and elastomeric polypeptide coating., Acta Biomater. 13 (2015) 111-120.

[155] C.M. Bellingham, M.A. Lillie, J.M. Gosline, G.M. Wright, B.C. Starcher, A.J. Bailey, et al., Recombinant human elastin polypeptides self-assemble into biomaterials with elastinlike properties, Biopolymers. 70 (2003) 445-455.

[156] E.M. Srokowski, K.A. Woodhouse, Development and characterisation of novel crosslinked bio-elastomeric materials, J. Biomater. Sci. Polym. Ed. 19 (2008) 785-799.

[157] L. Li, K.L. Kiick, M. Editors-in-Chief: Krzysztof, M. Martin, 9.06 - Resilin in the 
Engineering of Elastomeric Biomaterials, in: Polym. Sci. A Compr. Ref., Elsevier, Amsterdam, 2012: pp. 105-116.

[158] L. Li, K.L. Kiick, Resilin-Based Materials for Biomedical Applications, ACS Macro Lett. 2 (2013) 635-640.

[159] T. Weis-Fogh, A rubber-like protein in insect cuticles, J. Exp. Biol. 37 (1960) 889-907.

[160] S.N. Gorb, Serial elastic elements in the damselfly wing: Mobile vein joints contain resilin, Naturwissenschaften. 86 (1999) 552-555.

[161] M. Burrows, S.R. Shaw, G.P. Sutton, Resilin and chitinous cuticle form a composite structure for energy storage in jumping by froghopper insects, Bmc Biol. 6 (2008) 41-57.

[162] R.E. Lyons, D.C.C. Wong, M. Kim, N. Lekieffre, M.G. Huson, T. Vuocolo, et al., Molecular and functional characterisation of resilin across three insect orders., Insect Biochem. Mol. Biol. 41 (2011) 881-890.

[163] H. Bennet-Clark, The first description of resilin, J. Exp. Biol. 210 (2007) 3879-3881.

[164] R.S.C. Su, Y. Kim, J.C. Liu, Resilin: Protein-based elastomeric biomaterials, Acta Biomater. 10 (2014) 1601-1611.

[165] D.H. Ardell, S.O. Andersen, Tentative identification of a resilin gene in Drosophila melanogaster, Insect Biochem. Mol. Biol. 31 (2001) 965-970.

[166] C.M. Elvin, A.G. Carr, M.G. Huson, J.M. Maxwell, R.D. Pearson, T. Vuocolo, et al., Synthesis and properties of crosslinked recombinant pro-resilin, Nature. 437 (2005) 9991002.

[167] D. Young, H.C. Bennet-Clark, The role of the timbal in cicada sound production, J. Exp. Biol. 198 (1995) 1001-1019.

[168] H.C. Bennet-Clark, Tymbal mechanics and the control of song frequency in the cicada Cyclochila australasiae, J. Exp. Biol. 200 (1997) 1681-1694.

[169] P.J. Fonseca, H.C. Bennet-Clark, Asymmetry of tymbal action and structure in a cicada: A possible role in the production of complex songs, J. Exp. Biol. 201 (1998) 717-730.

[170] J.F. V Vincent, U.G.K. Wegst, Design and mechanical properties of insect cuticle, Arthropod Struct. Dev. 33 (2004) 187-199.

[171] F. Haas Gorb, S. and Wootton, R. J., F. Haas, S. Gorb, R.J. Wootton, Elastic joints in dermapteran hind wings: materials and wing folding, Arthropod Struct. Dev. 29 (2000) 137-146.

[172] F. Haas, S. Gorb, R. Blickhan, The function of resilin in beetle wings, Proc. R. Soc. London Ser. B-Biological Sci. 267 (2000) 1375-1381.

[173] T. Weis-Fogh, Molecular interpretation of elasticity of resilin, a rubber-like protein , J. Mol. Biol. 3 (1961) 648-667.

[174] K. Bailey, T. Weis-Fogh, Amino acid composition of a new rubber-like protein, resilin, Biochim. Biophys. Acta. 48 (1961) 452-459. 
[175] S.O. Andersen, T. Weis-Fogh, J.E.T. J.W.L. Beament, V.B. Wigglesworth, Resilin. A Rubberlike Protein in Arthropod Cuticle, in: Adv. In Insect Phys., Academic Press, 1964: pp. $1-65$.

[176] R.E. Lyons, E. Lesieur, M. Kim, D.C.C. Wong, M.G. Huson, K.M. Nairn, et al., Design and facile production of recombinant resilin-like polypeptides: gene construction and a rapid protein purification method, Protein Eng. Des. Sel. 20 (2007) 25-32.

[177] R.E. Lyons, K.M. Nairn, M.G. Huson, M. Kim, G. Dumsday, C.M. Elvin, Comparisons of Recombinant Resilin-like Proteins: Repetitive Domains Are Sufficient to Confer Resilinlike Properties, Biomacromolecules. 10 (2009) 3009-3014.

[178] J.N. Renner, K.M. Cherry, R.S.C. Su, J.C. Liu, Characterization of Resilin-Based Materials for Tissue Engineering Applications, Biomacromolecules. 13 (2012) 36783685 .

[179] G.K. Qin, A. Rivkin, S. Lapidot, X. Hu, I. Preis, S.B. Arinus, et al., Recombinant exonencoded resilins for elastomeric biomaterials, Biomaterials. 32 (2011) 9231-9243.

[180] S. Lv, D.M. Dudek, Y. Cao, M.M. Balamurali, J. Gosline, H.B. Li, Designed biomaterials to mimic the mechanical properties of muscles, Nature. 465 (2010) 69-73.

[181] C.L. McGann, R.E. Dumm, A.K. Jurusik, I. Sidhu, K.L. Kiick, Thiol-ene Photocrosslinking of Cytocompatible Resilin-Like Polypeptide-PEG Hydrogels, Macromol. Biosci. 16 (2016) 129-138.

[182] N.K. Dutta, M.Y. Truong, S. Mayavan, N.R. Choudhury, C.M. Elvin, M. Kim, et al., A Genetically Engineered Protein Responsive to Multiple Stimuli, Angew. ChemieInternational Ed. 50 (2011) 4428-4431.

[183] U. Hersel, C. Dahmen, H. Kessler, RGD modified polymers: biomaterials for stimulated cell adhesion and beyond, Biomaterials. 24 (2003) 4385-4415.

[184] H. Nagase, G.B. Fields, Human matrix metalloproteinase specificity studies using collagen sequence-based synthetic peptides, Biopolymers. 40 (1996) 399-416.

[185] M.P. Lutolf, J.L. Lauer-Fields, H.G. Schmoekel, A.T. Metters, F.E. Weber, G.B. Fields, et al., Synthetic matrix metalloproteinase-sensitive hydrogels for the conduction of tissue regeneration: engineering cell-invasion characteristics., Proc. Natl. Acad. Sci. U. S. A. 100 (2003) 5413-8.

[186] N. Yamaguchi, L. Zhang, B.S. Chae, C.S. Palla, E.M. Furst, K.L. Kiick, Growth factor mediated assembly of cell receptor-responsive hydrogels, J. Am. Chem. Soc. 129 (2007) 3040-3041.

[187] S.H. Kim, K.L. Kiick, Heparin-mimetic sulfated peptides with modulated affinities for heparin-binding peptides and growth factors, Peptides. 28 (2007) 2125-2136.

[188] M.B. Charati, J.L. Ifkovits, J.A. Burdick, J.G. Linhardt, K.L. Kiick, Hydrophilic elastomeric biomaterials based on resilin-like polypeptides, Soft Matter. 5 (2009) 34123416.

[189] L. Li, S. Teller, R.J. Clifton, X. Jia, K.L. Kiick, Tunable Mechanical Stability and 
Deformation Response of a Resilin-Based Elastomer, Biomacromolecules. 12 (2011) 2302-2310.

[190] T. Jiao, A. Farran, X. Jia, R.J. Clifton, High Frequency Measurements of Viscoelastic Properties of Hydrogels for Vocal Fold Regeneration, Exp. Mech. 49 (2009) 235-246.

[191] K.S. Straley, S.C. Heilshorn, Independent tuning of multiple biomaterial properties using protein engineering, Soft Matter. 5 (2009) 114-124.

[192] L. Li, Z. Tong, X. Jia, K.L. Kiick, Resilin-Like Polypeptide Hydrogels Engineered for Versatile Biological Functions., Soft Matter. 9 (2013) 665-673.

[193] L. Li, K. Kiick, Transient Dynamic Mechanical Analysis of Resilin-based Elastomeric Hydrogels, Front. Chem. 2 (2014) 20-32.

[194] L. Li, A. Mahara, Z. Tong, E.A. Levenson, C.L. McGann, X. Jia, et al., Recombinant Resilin-Based Bioelastomers for Regenerative Medicine Applications, Adv. Healthc. Mater. 5 (2016) 266-275.

[195] C.L. McGann, E.A. Levenson, K.L. Kiick, Resilin-Based Hybrid Hydrogels for Cardiovascular Tissue Engineering, Macromol. Chem. Phys. 214 (2013) 203-213.

[196] C.L. McGann, R.E. Akins, K.L. Kiick, Resilin-PEG Hybrid Hydrogels Yield Degradable Elastomeric Scaffolds with Heterogeneous Microstructure, Biomacromolecules. 17 (2016) $128-140$.

[197] H.K. Lau, L. Li, A.K. Jurusik, C.R. Sabanayagam, K.L. Kiick, Aqueous Liquid-Liquid Phase Separation of Resilin-like Polypeptide/Polyethylene Glycol Solutions for Formation of Microstructured Hydrogels, ACS Biomater. Sci. Eng. (2016). doi:10.1021/acsbiomaterials.6b00076.

[198] I.R. Titze, Mechanical stress in phonation, J. Voice. 8 (1994) 99-105.

[199] Y.B. Min, I.R. Titze, F. Alipour-Haghighi, Stress-Strain Response of the Human Vocal Ligament, Ann. Otol. Rhinol. Laryngol. 104 (1995) 563-569.

[200] I.R. Titze, S.A. Klemuk, S. Gray, Methodology for rheological testing of engineered biomaterials at low audio frequencies, J. Acoust. Soc. Am. 115 (2004) 392-401.

[201] Z. Tong, R.L. Duncan, X. Jia, Modulating the Behaviors of Mesenchymal Stem Cells Via the Combination of High-Frequency Vibratory Stimulations and Fibrous Scaffolds, Tissue Eng. Part A. 19 (2013) 1862-1878.

[202] J.C. Wolchok, C. Brokopp, C.J. Underwood, P.A. Tresco, The effect of bioreactor induced vibrational stimulation on extracellular matrix production from human derived fibroblasts, Biomaterials. 30 (2009) 327-335.

[203] I.R. Titze, R.W. Hitchcock, K. Broadhead, K. Webb, W. Li, S.D. Gray, et al., Design and validation of a bioreactor for engineering vocal fold tissues under combined tensile and vibrational stresses, J Biomech. 37 (2004) 1521-1529.

[204] J.K. Kutty, K. Webb, Vibration stimulates vocal mucosa-like matrix expression by hydrogel-encapsulated fibroblasts, J Tissue Eng Regen Med. 4 (2010) 62-72. 
[205] A.J. Farran, S.S. Teller, F. Jia, R.J. Clifton, R.L. Duncan, X. Jia, Design and characterization of a dynamic vibrational culture system, J Tissue Eng Regen Med. 7 (2013) 213-225.

[206] Z. Tong, A.B. Zerdoum, R.L. Duncan, X. Jia, Dynamic Vibration Cooperates with Connective Tissue Growth Factor to Modulate Stem Cell Behaviors, Tissue Eng. Part A. 20 (2014) 1922-1934.

[207] A.B. Zerdoum, Z. Tong, B. Bachman, X. Jia, Construction and characterization of a novel vocal fold bioreactor, J Vis Exp. (2014) e51594.

[208] S.A. Klemuk, S. Jaiswal, I.R. Titze, Cell viability viscoelastic measurement in a rheometer used to stress and engineer tissues at low sonic frequencies, J Acoust Soc Am. 124 (2008) 2330-2339.

[209] M.B. Christensen, J.C. Wolchok, S.A. Klemuk, I.R. Titze, Development of a bilayer ring system for achieving high strain in commercial rheometers, J Biomech. 48 (2015) 35123516.

[210] J. Gaston, B. Quinchia Rios, R. Bartlett, C. Berchtold, S.L. Thibeault, The response of vocal fold fibroblasts and mesenchymal stromal cells to vibration, PLoS One. 7 (2012) e30965.

[211] R.S. Bartlett, J.D. Gaston, T.Y. Yen, S. Ye, C. Kendziorski, S.L. Thibeault, Biomechanical Screening of Cell Therapies for Vocal Fold Scar, Tissue Eng Part A. 21 (2015) 2437-2447.

[212] I.R. Titze, K. Broadhead, P. Tresco, S. Gray, Strain distribution in an elastic substrate vibrated in a bioreactor for vocal fold tissue engineering, J Biomech. 38 (2005) 24062414. 